\title{
Impacts of wave spreading and multidirectional waves on estimating Stokes drift
}

\author{
A. Webb ${ }^{\mathrm{a}, *}$, B. Fox-Kemper ${ }^{\mathrm{b}}$ \\ ${ }^{a}$ Dept. of Ocean Technology, Policy, and Environment, The University of Tokyo, Japan \\ ${ }^{b}$ Dept. of Earth, Environmental, and Planetary Sciences, Brown University, Providence, \\ Rhode Island
}

\section{Abstract}

The Stokes drift, and its leading-order approximation, for a random sea depend upon the interaction of different wave groups and the process of wave spreading. Here Stokes drift direction and magnitude from prescribed spectra, local observational buoy data, and global model WAVEWATCH III output are used to analyze approximations of Stokes drift for directional random seas in deep water. To facilitate analysis, a new approximation is defined to incorporate the systematic effects of wave spreading. Stokes drift is typically overestimated by ignoring these effects or by ignoring directional differences in swell and wind seas. These two errors are differentiated and found to be largely uncorrelated. These errors depend strongly on depth, with deeper Stokes drift favoring narrow-banded swell and shallower Stokes drift favoring wind seas. Results are consistent among the data examined. Mean Stokes drift magnitude reductions from wave spreading and multidirectional wave

\footnotetext{
*Corresponding author

Email addresses: adrean.webb@gmail.com (A. Webb), baylor@brown.edu (B. Fox-Kemper)

$U R L:$ http://www. adreanwebb.com (A. Webb), http://www.fox-kemper.com (B. Fox-Kemper)
} 
effects alone are $14-20 \%$ and $7-23 \%$ respectively, giving a combined reduction of $20-40 \%$ versus unidirectional waves, depending on wave age and depth. Approximations that do not include these reductions however, will on average overestimate Stokes drift by $16-26 \%, 26-43 \%$, and $45-71 \%$ respectively. In addition to magnitude, the direction of Stokes drift is also affected and multidirectional waves generate a directional veer with depth: the 30/60/90\% confidence intervals are bounded (approximately) by $\pm 0.12 / 0.28 / 0.84$ radians $( \pm 7 / 16 / 48 \mathrm{deg})$ at the surface, with smaller intervals at depth. Complementary depth-integrated approximations are also investigated and directional effects are similar with depth-dependent subsurface results. Furthermore, an optimized directional spread correction for the surface is nearly identical for global simulations and a buoy located at Ocean Weather Station $\mathrm{P}\left(50^{\circ} \mathrm{N}\right.$ $\left.145^{\circ} \mathrm{W}\right)$, and does not require directional wave spectrum data.

Keywords: Stokes drift, unidirectional waves, wave spreading

\section{1. Introduction}

2 Stokes drift is an important quantity in categorizing the effects of waves 3 generally, but ocean surface gravity waves in particular. Stokes drift quan-

4 tifies the forcing of wave-driven upper ocean mixing (Craik and Leibovich, 5 1976; Thorpe, 1985; D'Asaro, 2014; D'Asaro et al., 2014), is crucial to under6 standing the open ocean interaction between waves and mean flows 7 (McWilliams et al., 2012; McWilliams and Fox-Kemper, 2013), and is signif8 icant in understanding open ocean sea surface transport (McWilliams and 9 Restrepo, 1999; Ardhuin et al., 2009; Sullivan and McWilliams, 2010; Röhrs 10 et al., 2014). Interest in parameterizing the effects of wave-driven mixing in 
global climate models has spurred an interest in parameterizations relating Stokes drift and other wave parameters to mixing (Kantha and Clayson, 2004; Harcourt and D'Asaro, 2008; Babanin and Haus, 2009; Grant and Belcher, 2009; Huang et al., 2011; Van Roekel et al., 2012; Belcher et al., 2012; Harcourt, 2013; Fan and Griffies, 2014) and climate impacts generally (Cavaleri et al., 2012).

It is often overlooked that the depth-dependent and depth-integrated Stokes drifts are not easily measured or generated from wind and wave data. To simplify, an unidirectional seas assumption, in which all the waves are moving in the same direction, is commonly used in practice. Kenyon (1969) was the first to infer this unidirectional Stokes drift from the wave spectrum, ${ }^{1}$ and Dalrymple and Dean (1984) provide a formulation of the depthintegrated Stokes drift. McWilliams and Restrepo (1999) chose to infer the unidirectional Stokes drift from wind data, not trusting the wave observation or modeling of the time. While some wave parameters are easily measured even by satellite (Gommenginger et al., 2003), Webb and Fox-Kemper (2011) show that it is difficult to accurately measure the higher moments of the wave spectrum needed to estimate surface or subsurface unidirectional Stokes drift, with about half of the error stemming from amplitude errors and half from frequency errors. Nonetheless, Webb and Fox-Kemper (2011) find that the model to model discrepancy in depth-varying unidirectional Stokes drift is of a similar magnitude to the model to satellite discrepancy. Since the depth-integrated unidirectional Stokes drift depends primarily on lower

\footnotetext{
${ }^{1}$ See Section 1.1 for a full definition.
} 
spectral moments (Myrhaug, 2013a), it does not amplify frequency errors so profoundly. Care is required however in evaluating errors in the nonlinear functions that relate wave fields to the surface and depth-integrated unidirectional Stokes drift, and using conditional probabilities is a recent advance (Myrhaug, 2013b,a; Myrhaug et al., 2014). Recently several papers have appeared that are closely related to this one; Liu et al. (2014), Carrasco et al. (2014), and Breivik et al. (2014) discuss similar issues to those addressed here in passing.

\subsection{Stokes drift for directional and unidirectional random seas}

Before proceeding further, it is prudent to briefly review the definitions of Stokes drift for directional and unidirectional random seas. Here we will follow and build upon notation from Webb and Fox-Kemper (2011) and Webb (2013). In addition, a list of common abbreviations are given in Table 1 for quick reference.

The directional random sea approximation is a power spectral density estimate derived from the leading-order Stokes drift velocity using smallamplitude linear surface gravity waves. Using directional-frequency spectra, $\mathcal{S}_{f \theta}$, and the deep-water dispersion relation, ${ }^{2}$ a cell-averaged estimate can be written as

$$
\begin{aligned}
\boldsymbol{u}_{2 D_{h}}^{S} & =\boldsymbol{u}_{2 D_{h}}^{S}\left(\boldsymbol{x}, t ; \boldsymbol{L}_{h}, T\right) \\
& \approx \frac{16 \pi^{3}}{g} \int_{0}^{\infty} \int_{-\pi}^{\pi}(\cos \theta, \sin \theta, 0) f^{3} \mathcal{S}_{f \theta}(f, \theta) \exp \left[\frac{8 \pi^{2} f^{2} z}{g}\right] d \theta d f
\end{aligned}
$$

\footnotetext{
${ }^{2}$ The deep-water dispersion relation is used for consistency with previous work.
} 
where $\boldsymbol{L}_{h}$ and $T$ represent the implicit horizontal length and time scales necessary to resolve the discrete spectra. To be precise, $\boldsymbol{x}=\left(\boldsymbol{x}_{h}, z\right)$ is a generic horizontal cell center with an arbitrary depth. In addition, (1) is typically valid for $L_{\min }>1 \mathrm{~km}$ and $T \gg \sqrt{2 L_{\max } / \pi \mathrm{g}}$; these dimensions ensure scale separation from the wavelength and period of typical surface gravity waves. ${ }^{3}$ This horizontally-two-dimensional Stokes drift vector uses 2D directionalfrequency spectra and is termed $2 D_{h}-S D$ to distinguish it from from later horizontally-one-dimensional approximations that use 1D frequency spectra. Due to a limitation in directional-frequency spectral data from both observations and models, the following unidirectional wave assumption is often used to simplify calculations of Stokes drift:

$$
\mathcal{S}_{f \theta}(f, \theta)=\delta\left(\theta-\theta^{\prime}\right) \mathcal{S}_{f}(f)
$$

Here, $\delta$ is the Dirac delta function and $\theta^{\prime}$ is used to define the assumed Stokes drift direction as $\hat{\boldsymbol{e}}^{\mathrm{w}}=\left(\cos \theta^{\prime}, \sin \theta^{\prime}, 0\right)$. This simplifies the interior integral in (1) as

$$
\int_{-\pi}^{\pi}(\cos \theta, \sin \theta, 0) \mathcal{S}_{f \theta}(f, \theta) d \theta=\hat{\boldsymbol{e}}^{\mathrm{w}} \mathcal{S}_{f}(f),
$$

and results in a simpler horizontally-one-dimensional form of Stokes drift (henceforth $1 D_{h}-S D$ ) and its surface value, given as:

$$
\begin{aligned}
\boldsymbol{u}_{1 \mathrm{D}_{h}}^{S} & =\hat{\boldsymbol{e}}^{\mathrm{w}} \frac{16 \pi^{3}}{\mathrm{~g}} \int_{0}^{\infty} f^{3} S_{f}(f) \exp \left[\frac{8 \pi^{2} f^{2} z}{\mathrm{~g}}\right] d f \\
\left.\boldsymbol{u}_{1 \mathrm{D}_{h}}^{S}\right|_{z=0} & =\hat{\boldsymbol{e}}^{\mathrm{w}} \frac{16 \pi^{3}}{\mathrm{~g}} \int_{0}^{\infty} f^{3} S_{f}(f) d f=\hat{\boldsymbol{e}}^{\mathrm{w}} \frac{16 \pi^{3} m_{3}}{\mathrm{~g}} .
\end{aligned}
$$

\footnotetext{
${ }^{3} L_{\min }$ and $L_{\max }$ simply refer to the minimum and maximum horizontal dimensions for a rectangular grid cell.
} 
Notice now that only the third moment of the $1 \mathrm{D}$ wave spectrum, $m_{3}$, is required to estimate the surface $1 \mathrm{D}_{h}-\mathrm{SD}$.

\subsection{Motivation for current study}

While the $1 \mathrm{D}_{h}$-SD approximation is commonly used in literature, the unidirectional seas assumption is a strong one that affects both the magnitude and direction of Stokes drift. Without access to the full 2D wave spectra however, estimating errors in any calculation poses a challenge since the degree of wave spreading is unknown and the presence of multiple wave groups can be hidden. These features affect both the magnitude and direction in a nonlinear fashion that is sensitive to depth. By assumption, the $1 \mathrm{D}_{h}$-SD approximation ignores these features and as a result overestimates the magnitude. In addition, the assumed direction (usually the wind seas direction) is often misaligned with the actual direction. These differences can be substantial and are not readily quantifiable; such a quantification is the primary purpose here.

Despite this, the $1 \mathrm{D}_{h}$-SD approximation has often been preferable since it simplifies calculations and access to 2D spectral data has been historically limited. Recently however, there has been a trend toward using the $2 \mathrm{D}_{h}$-SD approximation for calculations that are sensitive to Stokes drift despite the exponential increase in computation. These depth-dependent values can be calculated for wave parameterizations online as needed without increasing storage or using an assumed profile. ${ }^{4}$ While this is appropriate and rec-

\footnotetext{
${ }^{4}$ An exponentially decaying profile can sometimes be inaccurate. See Fig. 4 for an example.
} 
ommended, the missing physics are unclear and comparisons of calculations using $1 \mathrm{D}_{h}$-SD and $2 \mathrm{D}_{h}$-SD approximations, as well as other intermediate approximations, are needed.

\subsection{Overview of current study}

In this paper, a first attempt has been made to better understand and quantify the influence of wave spreading and multidirectional waves on Stokes drift. While the former is essentially a stochastic process that is not sensitive to local or remote forcing conditions, the latter is strongly dependent on the interaction of different wave groups, particularly wind seas (local) and swell waves (remotely-generated). To achieve this, the Stokes drift estimation methods of Webb and Fox-Kemper (2011) are expanded to include directional information about the wave field. Again, prescribed wave spectra and global WAVEWATCH III simulations (version 2.22; Tolman, 2002) are used as a reference and model basis (respectively), while a deep-ocean moored buoy located at Ocean Weather Station $\mathrm{P}\left(50^{\circ} \mathrm{N} 145^{\circ} \mathrm{W}\right)$ is used as a directionalwave-information observational dataset (Thomson et al., 2013; Belka et al., 2014)..$^{5}$ In addition, a new $1 \mathrm{D}_{h}$-SD approximation is introduced to quantify and differentiate these directional effects on Stokes drift.

In Section 2, several examples are selected to illustrate different directional effects in the random seas Stokes drift. In Section 3, differences be-

\footnotetext{
${ }^{5}$ It should be noted that Rascle and Ardhuin (2013) find sensitivity in Stokes drift with parameterizations used, so this work ought to be repeated with different versions of the model for robustness. However in this paper, our results are less sensitive since we only examine Stokes drift ratios. In addition, we find good agreement among comparisons so
} WAVEWATCH III simulations analyzed here cannot be grossly incorrect. 
tween $1 \mathrm{D}_{h}$-SD and $2 \mathrm{D}_{h}$-SD are formally explored and a new $1 \mathrm{D}_{h}$-SD approximation is developed to include a form of wave spreading advanced by Donelan et al. (1985). In Sections 4-6, the directional effects of wave spreading and multidirectional waves on the depth-dependent and depth-integrated Stokes drift are analyzed using prescribed wave spectra, observations, and model simulations. Finally, a treatment of error is given in Section 7 and results are discussed and summarized in Sections 8 and 9 respectively.

\section{Pitfalls of the unidirectional seas assumption}

Several simple examples are given below to illustrate some of the challenges of using 1D wave spectra to calculate Stokes drift. The last example will provide some insight on how the $1 \mathrm{D}_{h}$-SD approximation can be improved.

\subsection{Multidirectional waves and Stokes drift magnitude}

When multiple wave groups are present, the magnitude of Stokes drift will depend on the angle of incidence between them. To illustrate, consider two monochromatic waves passing through the same point from different directions (see Fig. 1). For simplicity, let the peak frequency and amplitude of the waves be the same, and suppose that their phase varies slowly and sufficiently so that interference patterns are not persistent. First recall that a monochromatic spectrum for a peak frequency $f_{p}$, wave amplitude $a$, and direction $\tilde{\theta}$, can be defined as

$$
\mathcal{S}_{\text {mono }, f \theta}(f, \theta)=\frac{a^{2}}{2} \delta\left(f-f_{p}\right) \delta(\theta-\tilde{\theta}) .
$$

Using the $2 \mathrm{D}_{h}$-SD approximation, the resulting Stokes drift for this wave is

$$
\left.\boldsymbol{u}_{2 \mathrm{D}_{h}, \text { mono }}^{S}\right|_{\boldsymbol{x}_{0}}=(\cos \tilde{\theta}, \sin \tilde{\theta}, 0) \frac{8 \pi^{3} a^{2} f_{p}^{3}}{\mathrm{~g}} \exp \left[\frac{8 \pi^{2} f_{p}^{2} z_{0}}{\mathrm{~g}}\right] \text {. }
$$



${ }_{145} \sum_{i=1}^{n} \frac{a_{i}^{2}}{2} \delta\left(f-f_{i}\right) \delta\left(\theta-\theta_{i}\right)$, as

$$
\left.\boldsymbol{u}_{n \text {-chro }}^{S}\right|_{\boldsymbol{x}_{0}}=\sum_{i=1}^{n}\left(\cos \theta_{i}, \sin \theta_{i}, 0\right) A_{i}\left(z_{0}\right)
$$

and the resulting Stokes drift is then can be shown that $\left.\boldsymbol{u}_{1 \mathrm{D}_{h}, \text { bi }}^{S}\right|_{\boldsymbol{x}_{0}}=\left.2 \boldsymbol{u}_{2 \mathrm{D}_{h} \text {,mono }}^{S}\right|_{\boldsymbol{x}_{0}}$ and hence

$$
\left.\boldsymbol{u}_{2 D_{h}, \mathrm{bi}}^{S}\right|_{\boldsymbol{x}_{0}}=\left.\cos \theta^{\prime} \boldsymbol{u}_{1 D_{h}, \mathrm{bi}}^{S}\right|_{\boldsymbol{x}_{0}}
$$
superposition of Stokes drift (see Webb and Fox-Kemper, 2011).

\subsection{Multidirectional waves and Stokes drift direction}

$$
\sum_{i=1} \frac{1}{2} \delta\left(f-f_{i}\right) \delta\left(\theta-\theta_{i}\right) \text {, as }
$$

$$
\begin{aligned}
\left.\boldsymbol{u}_{2 \mathrm{D}_{h}, \text { bi }}^{S}\right|_{\boldsymbol{x}_{0}}= & \left(\cos \left(\bar{\theta}+\theta^{\prime}\right)+\cos \left(\bar{\theta}-\theta^{\prime}\right), \sin \left(\bar{\theta}+\theta^{\prime}\right)+\sin \left(\bar{\theta}-\theta^{\prime}\right), 0\right) \\
& \times \frac{8 \pi^{3} a^{2} f_{p}^{3}}{\mathrm{~g}} \exp \left[\frac{8 \pi^{2} f_{p}^{2} z_{0}}{\mathrm{~g}}\right] \\
= & \left.2 \cos \theta^{\prime} \boldsymbol{u}_{2 \mathrm{D}_{h}, \text { mono }}^{S}\right|_{\boldsymbol{x}_{0}} .
\end{aligned}
$$

This is analogous to comparing $1 \mathrm{D}_{h}-\mathrm{SD}$ and $2 \mathrm{D}_{h}$-SD approximations and it

This implies that in general, the $1 \mathrm{D}_{h}$-SD approximation will overestimate Stokes drift whenever both wind seas and swell (or other multidirectional waves) are present. Note that to the order of approximation considered here, linear superposition of the waves is assumed to continue to hold for linear

In the previous example, the resulting Stokes drift magnitude follows simple vector addition. This generalizes for any $n$-chromatic wave, $\mathcal{S}_{n \text {-chro, } f \theta}=$ 
where $A_{i}(z)=\frac{8 \pi^{3} a_{i}^{2} f_{i}^{3}}{\mathrm{~g}} \exp \left[\frac{8 \pi^{2} f_{i}^{2} z}{\mathrm{~g}}\right]$ for some amplitude and peak frequency $a_{i}$ and $f_{i}$. Again for simplicity, consider a bichromatic wave but with different peak frequencies such that $a_{1}=a_{2}, \theta_{1} \neq \theta_{2}$ and $f_{2}=e^{1} f_{1}$. Then for the depth

$$
z=\frac{-3 \mathrm{~g}}{8 \pi^{2}\left(e^{2}-1\right) f_{1}^{2}} \approx \frac{-0.058}{f_{1}^{2}},
$$

the Stokes drift direction will be the directional average of both waves, $\left(\theta_{1}+\theta_{2}\right) / 2$. However, for any depth above this value, the dominant Stokes drift direction will be directed more toward the higher-frequency monochromatic wave. Likewise, for any depth below this value, the direction will be oriented more towards the lower frequency wave. We will refer to this effect as veer (to distinguish it from the directional magnitude loss in the previous section) and this will be discussed in more detail later. However in general, the direction of Stokes drift should be expected to align with higher frequency, shorter wavelength wind seas at the surface and lower frequency, longer wavelength swell at greater depths, whenever both are present.

\subsection{Wave spreading and Stokes drift magnitude}

In addition to multidirectional waves, the degree of wave spreading also contributes to directional magnitude loss in Stokes drift. To illustrate, consider the following modification to the unidirectional spectra in (2) (shown here again):

$$
\begin{aligned}
& \mathcal{S}_{1, f \theta}(f, \theta)=\sqrt{\frac{2}{\pi}} \exp \left[-2 \theta^{2}\right] \mathcal{S}_{f}(f), \\
& \mathcal{S}_{2, f \theta}(f, \theta)=\delta(\theta) \mathcal{S}_{f}(f) .
\end{aligned}
$$


Notice that both directional-frequency spectra are normalized to have the same frequency spectra after integrating in $\theta$ and are directed on average in the $\hat{e}_{1}$ direction. However due to wave spreading, the magnitudes are not equivalent and $\left\|\boldsymbol{u}_{1}^{S}\right\| \approx 0.882\left\|\boldsymbol{u}_{2}^{S}\right\|$. The $\|\cdot\|$ notation is defined here (and throughout) as the Euclidian norm.

In all three examples, the magnitude and/or direction of Stokes drift is affected by the use of directionally-averaged $1 \mathrm{D}$ wave spectra. In the first two examples, the differences between $1 \mathrm{D}_{h^{-}}$-SD and $2 \mathrm{D}_{h}$-SD are dependent upon properties of coincidences of a random sea (e.g., how often are the waves multidirectional, what are typical frequency and directional differences, etc.). In this example however, the differences are systematic (i.e., determined by the wave spreading function) and not a coincidental process. It will be later shown that the error due to wave spreading in $1 \mathrm{D}_{h}$-SD approximations can be removed if the frequency-dependent directional spread is known.

\section{An improved $1 \mathrm{D}_{h}$-SD estimate}

To aid understanding, formal relations between the $1 \mathrm{D}_{h^{-}}$SD and $2 \mathrm{D}_{h^{-}}$ SD approximations are defined and utilized in this section to develop an improved $1 \mathrm{D}_{h}$-SD approximation. Incorporating previous work (Webb and Fox-Kemper, 2011, Appendices A.3 and A.4), this new approximation uses an empirical spreading function to include the effects of wave spreading and is used in later sections to differentiate between directional magnitude losses in Stokes drift due to wave spreading and multidirectional waves. 


\subsection{Defining a generic directional distribution}

The 1D frequency spectrum is defined such that

$$
\mathcal{S}_{f}(f)=\int_{-\pi}^{\pi} \mathcal{S}_{f \theta}(f, \theta) d \theta .
$$

This is equivalent to splitting the directional-frequency spectrum into a frequency component $\left(\mathcal{S}_{f}\right)$ and a directional distribution $(D)$ and integrating in direction $(\theta)$. For a fixed frequency $f_{\alpha}$, a fixed directional distribution can be defined as

$$
D_{f_{\alpha}}(\theta)=\left\{\begin{array}{cc}
\frac{\mathcal{S}_{f \theta}\left(f_{\alpha}, \theta\right)}{\int_{-\pi}^{\pi} \mathcal{S}_{f \theta}\left(f_{\alpha}, \theta\right) d \theta}, & \mathcal{S}_{f \theta}\left(f_{\alpha}, \theta\right) \neq 0 \\
\frac{1}{2 \pi}, & \mathcal{S}_{f \theta}\left(f_{\alpha}, \theta\right)=0
\end{array}\right.
$$

Assuming $D$ can be defined continuously in $f\left(\right.$ as $D(f, \theta) \equiv D_{f}(\theta)$ ), the directional distribution has the property

$$
\int_{-\pi}^{\pi} D(f, \theta) d \theta=1
$$

for every $f$, and the directional-frequency and frequency spectrum can be related by

$$
\int_{0}^{\infty} \int_{-\pi}^{\pi} \mathcal{S}_{f \theta}(f, \theta) d \theta d f=\int_{0}^{\infty} \int_{-\pi}^{\pi} D(f, \theta) \mathcal{S}_{f}(f) d \theta d f=\int_{0}^{\infty} \mathcal{S}_{f}(f) d f .
$$

\subsection{Defining a generic directional component}

Due to the vector component $(\cos \theta, \sin \theta, 0)$ in the integrand, the behavior of Stokes drift is not always intuitive. To identify the role it plays more concretely, let the combined integral of any generic directional distribution and vector component, or

$$
\boldsymbol{H}(f)=\int_{-\pi}^{\pi}(\cos \theta, \sin \theta, 0) D(f, \theta) d \theta
$$


be termed the directional-Stokes-drift-component. ${ }^{6}$ Then the $2 \mathrm{D}_{h}$-SD can be rewritten as

$$
\boldsymbol{u}_{2 D_{h}}^{S}=\frac{16 \pi^{3}}{g} \int_{0}^{\infty} \boldsymbol{H}(f) f^{3} S_{f}(f) \exp \left[\frac{8 \pi^{2} f^{2}}{g} z\right] d f
$$

Notice that for all unidirectional waves (i.e., $\mathcal{S}_{f, \theta}(f, \theta)=\delta(\theta-\bar{\theta}) \mathcal{S}_{f}(f)$ ), $\|\boldsymbol{H}(f)\|=1$ for all $f \in \mathbb{R}_{+}$. In this context, the $1 \mathrm{D}_{h}$-SD can be thought of as a $2 \mathrm{D}_{h}$-SD approximation with $\left\|\boldsymbol{H}_{1 \mathrm{D}_{h}}\right\|=1$ and $\boldsymbol{H}_{1 \mathrm{D}_{h}} /\left\|\boldsymbol{H}_{1 \mathrm{D}_{h}}\right\|=$ $(\cos \bar{\theta}, \sin \bar{\theta}, 0)$ for some assumed direction $\bar{\theta}$. The directional-Stokes-driftcomponent can now be used to quantify the directional magnitude loss due to wave spreading alone.

\subsection{The DHH wave spreading function}

There have been numerous studies on the topic of directional wave spreading and several well-known spreading functions proposed by Mitsuyasu et al. (1975), Hasselmann et al. (1980), and Donelan et al. (1985) were considered for the directional-Stokes-drift-component. In each study, an assumed parametric form was used with observational wave spectra to derive an empirical function for fetch-limited conditions in deep water. ${ }^{7}$ Mitsuyasu et al. (1975) and Hasselmann et al. (1980) used pitch-roll-heave buoys to measure the wave field whereas Donelan et al. (1985) used a spatial array of 14

\footnotetext{
${ }^{6}$ This was previously referred to as directional spread loss in Webb and Fox-Kemper (2011).

${ }^{7}$ Since these functions have been parametrized for fetch-limited conditions, they are likely to be inaccurate for fully-developed or even mixed sea conditions (Alves and Melo, 1999). However, the authors believe they are good candidates for the purposes of this initial analysis.
} 
wave staffs (Young, 2010). Of these three studies, the Donelan-Hamilton-Hui (henceforth abbreviated $\mathrm{DHH}$ ) field campaign had the highest spectral resolution and subsequent (observational and numerical) analyses have found better agreement with the DHH wave spreading function (Young, 1994; Forristall and Ewans, 1998; Ewans, 1998).

For these reasons and others to be discussed later, the DHH wave spreading function has been chosen for the directional-Stokes-drift-component. However it has been slightly modified here; the amplitude and domain have been adjusted to be normal and periodic in $\theta$ (Webb and Fox-Kemper, 2011) as

$$
\begin{aligned}
& D_{\text {DHH }}\left(f, \theta ; f_{p}, \bar{\theta}\right)=\frac{\beta\left(f / f_{p}\right)}{2 \tanh \left[\beta\left(f / f_{p}\right) \pi\right]} \\
& \quad \times \begin{cases}\operatorname{sech}^{2}\left[\beta\left(f / f_{p}\right) \theta\right], & \bar{\theta}=0, \\
\operatorname{sech}^{2}\left[\beta\left(f / f_{p}\right)(\theta-\bar{\theta})\right], & -\pi+|\bar{\theta}| \leq \bar{s} \theta \leq \pi, \\
\operatorname{sech}^{2}\left[\beta\left(f / f_{p}\right)(\theta-\bar{\theta}+2 \pi \bar{s})\right], & -\pi \leq \bar{s} \theta \leq-\pi+|\bar{\theta}| .\end{cases}
\end{aligned}
$$

Here, $f_{p}$ is the peak frequency, $\bar{\theta}(f) \in[-\pi, \pi]$ is the mean direction for a particular $f, \bar{s}$ is the sign of $\bar{\theta}$, and $\beta$ is given by

$$
\beta\left(f / f_{p}\right)= \begin{cases}2.61\left(f / f_{p}\right)^{1.3}, & 0.56<f / f_{p} \leq 0.95 \\ 2.28\left(f / f_{p}\right)^{-1.3}, & 0.95<f / f_{p}<1.6, \\ 1.24, & \text { otherwise. }\end{cases}
$$

In addition, it should be noted that $\beta$ is only piecewise continuous.

\subsection{The DHH directional-Stokes-drift-component}

Unfortunately, calculating the directional-Stokes-drift-component for the DHH wave spreading function is fairly complicated and requires the use of hypergeometric functions with complex arguments (Luke, 1969). To simplify, 
a Padé approximate of order [2/2] (Bender and Orszag, 1978) has been used to approximate the exact solution within a relative error of $3 \times 10^{-3}$, given by

$$
\begin{aligned}
\boldsymbol{H}_{\mathrm{DHH}}\left(f ; f_{p}, \bar{\theta}\right)=(\cos \bar{\theta}, \sin \bar{\theta}, 0) \\
\\
\times \begin{cases}\frac{0.52 f_{p}^{2}-3.3 f_{p} f+8.9 f^{2}}{f_{p}^{2}-3.4 f_{p} f+8.9 f^{2}}, & 0.56<f / f_{p} \leq 0.95, \\
\frac{0.98 f_{p}^{2}-0.19 f_{p} f+0.0058 f^{2}}{f_{p}^{2}-0.26 f_{p} f+0.12 f^{2}}, & 0.95<f / f_{p}<1.6, \\
0.777, & \text { otherwise. }\end{cases}
\end{aligned}
$$

Notice that the DHH directional-Stokes-drift-component requires knowledge of the peak frequency and the mean direction and is still a function in terms of frequency (i.e., the mean direction is not necessarily constant for all frequencies). While not the focus of this paper, this approximation is significantly faster computationally than using (19) to solve (17), requiring about the same number of multiplications and additions but no special function calls or numerical integration.

The magnitude of the DHH directional-Stokes-drift-component is depicted in Fig. 2. Notice that it is bounded as $0.777 \leq\left\|\boldsymbol{H}_{\mathrm{DHH}}\right\| \leq 0.934$. This implies $1 \mathrm{D}_{h}$-SD approximations may be overestimating $2 \mathrm{D}_{h}-\mathrm{SD}$ by as much as $30 \%$ due to wave spreading alone (since $\max \left\{\left\|\boldsymbol{H}_{1 \mathrm{D}_{h}}\right\| /\left\|\boldsymbol{H}_{D H H}\right\|\right\} \approx 1.287$ ).

\subsection{The new $1 D_{h}-D H H-S D$ approximation}

As mentioned in the example in Section 2.3 , the $1 \mathrm{D}_{h}$-SD approximation can be improved by using a wave spreading function to approximate the full 2D spectra for Stokes drift calculations. Here, the Padé-approximated, DHH directional-Stokes-drift-component, (21), will be used to modify (4) as

$$
\boldsymbol{u}_{\mathrm{DHH}}^{S}=\hat{\boldsymbol{e}}^{\mathrm{w}} \frac{16 \pi^{3}}{\mathrm{~g}} \int_{0}^{\infty} H_{\mathrm{DHH}}\left(f / f_{p}\right) f^{3} S_{f}(f) \exp \left[\frac{8 \pi^{2} f^{2} z}{\mathrm{~g}}\right] d f
$$


where $H_{\mathrm{DHH}}\left(f / f_{p}\right)=\left\|\boldsymbol{H}_{\mathrm{DHH}}\left(f ; f_{p}, \bar{\theta}=\hat{\boldsymbol{e}}^{\mathrm{w}}\right)\right\|$ and $\hat{\boldsymbol{e}}^{\mathrm{w}}$ is the chosen mean direction. The approximation requires knowledge of the peak frequency but this is fairly straight-forward to calculate in a model implementation. As with any $1 \mathrm{D}_{h}$-SD approximation, the choice of $\hat{\boldsymbol{e}}^{\mathrm{w}}$ is not always clear and will be discussed in Section 5. The improved $1 \mathrm{D}_{h}$ estimate will be termed $1 D_{h}-D H H-S D$ to distinguish it from the unidirectional approximation $\left(1 \mathrm{D}_{h^{-}}\right.$ $\mathrm{SD})$. As previously pointed out, there are multiple spreading functions and hence, the improved estimate is not limited to the use of the DHH directionalStokes-drift-component. While rarely used here, an alternative directionalStokes-drift-component (also displayed in Fig. 2) is derived in Appendix A and is based on a modification to the DHH wave spreading function proposed by Banner (1990) (henceforth abbreviated DHH-B).

\section{Depth-dependent Stokes drift magnitude}

Here, the $1 \mathrm{D}_{h}$-DHH-SD will be used with prescribed wave spectra (i.e., empirically formulated), observations, and model output to differentiate the directional magnitude losses in Stokes drift due to wave spreading and multidirectional waves. For surface Stokes drift magnitudes, it will be shown that estimates from all three types are in close agreement and that it is possible to correct $1 \mathrm{D}_{h}$-SD for wave spreading by multiplying by a constant.

\subsection{Analysis using $1 D_{h}-D H H-S D$ with prescribed wave spectra}

Here, spectra from three different empirically-derived formulas will be used to examine the role of wave spreading. Since prescribed spectra require knowledge of the peak frequency, e-folding depths will be used instead of 
depth to remove the peak frequency dependence and analyze the results. Let $n \in \mathbb{R}_{+}$and $z_{n}=-n \mathrm{~g} /\left(8 \pi^{2} f_{p}^{2}\right)$. Then for a monochromatic wave,

$$
\left\|\boldsymbol{u}_{\text {mono }}^{S}\left(z_{n}\right)\right\|=e^{-n}\left\|\boldsymbol{u}_{\text {mono }}^{S}(z=0)\right\| .
$$

In Table 2, three different peak frequencies are chosen (from high to low) to illustrate a typical range of e-folding depths. Notice that at each $e$-folding depth, the depth for the lowest frequency is approximately 50 times larger than the highest. This will aid an intuitive understanding shortly.

In addition to JONSWAP (Hasselmann et al., 1976) and Pierson and Moskowitz (1964, PM hereafter) prescribed spectra, empirical spectral shapes from Donelan et al. (1985) (abbreviated DHH as well) are also used to analyze the effects of wave spreading. Fetch-limited (wave age of 0.25 ) and fully-developed (wave age of 1.2) cases are chosen for the DHH spectra to mirror JONSWAP and PM shapes respectively. See Webb and Fox-Kemper (2011) for details and a visual comparison of the spectra.

To examine the reduction in Stokes drift magnitude due to wave spreading, ratios of $1 \mathrm{D}_{h}$-DHH-SD to $1 \mathrm{D}_{h}$-SD for $e$-folding depths are presented in Table 3 (for select values) and in Fig. 3. For the prescribed spectra selected, there is approximately a 8-20\% directional magnitude loss when using $1 \mathrm{D}_{h^{-}}$ DHH-SD instead of $1 \mathrm{D}_{h}$-SD. This loss is greatest for all spectra at the surface and flattens out at an $e$-folding depth near 3 before slowly increasing again. This is expected since initially the exponential component of Stokes drift will filter mostly higher frequencies as depth is increased (see Table 2). Eventually however, the majority of Stokes drift will be comprised of spectra with frequencies less than the peak and the magnitude loss will asymptotically increase towards $22 \%$. 


\subsection{Analysis using $1 D_{h}-D H H-S D$ with observational data}

Since interesting comparisons with $2 \mathrm{D}_{h}$-SD are not possible with prescribed spectra (as it is the superposition of different wave packets from different sources that leads to an interesting two-dimensional wave spectrum), it is necessary to use real or realistic data to study $2 \mathrm{D}$ spectrum effects on Stokes drift. Wave buoys have commonly been used to measure vertical velocities to infer the frequency spectra at a point (Holthuijsen, 2007). It is also possible for some buoys to estimate wave direction and thus infer the directional-frequency spectra. Care is needed however in interpreting this inferred spectra since an array of buoys would be needed to measure the full $2 \mathrm{D}$ spectra.

Here, observational data from Ocean Weather Station $\mathrm{P}$ (henceforth OWSP), a CDIP ${ }^{8}$ Datawell directional buoy (O'Reilly et al., 1996) stationed in deep water in the northeastern Pacific Ocean $\left(50^{\circ} \mathrm{N}, 145^{\circ} \mathrm{W}\right)$, is used to compare $1 \mathrm{D}_{h}$-SD, $1 \mathrm{D}_{h}$-DHH-SD, and $2 \mathrm{D}_{h}$-SD approximations. A continuous 27 month period, 30 minute intervals from 2010/6/21 to 2012/9/20, has been selected for comparison. Processed data (obtained from http: //cdip.ucsd.edu) is comprised of 1D frequency spectra with 64 frequency bands (with an initial and cutoff frequency of $0.025 \mathrm{~Hz}$ and $0.58 \mathrm{~Hz}$ respectively) and the first four non-constant Fourier coefficients of the directional distribution (for each frequency band). ${ }^{9}$ The directional-frequency spectra is generated using the Maximum Entropy Method (henceforth MEM; Lygre

\footnotetext{
${ }^{8}$ Coastal Data Information Program, Scripps Institution of Oceanography.

${ }^{9} \mathrm{By}$ definition, the first complex coefficient of the directional distribution is a constant $\left(c_{0}=1 / 2 \pi\right)$.
} 
and Krogstad, 1986) with a 9 deg directional discretization, chosen both to minimize abrupt transitions between the frequency bins and to use a similar discretization ratio $(\Delta f / \Delta \theta)$ as WAVEWATCH III (see Fig. $4 \mathrm{~b}$ for an example of the MEM-generated directional distribution). In addition, all approximations assume a spectral $f^{-5}$ tail (also for later comparison with WAVEWATCH III output).

Casual inspection of the 2D spectral data shows that both wave spreading and multidirectional waves are naturally present. As a result, occasional differences between the approximations are strikingly noticeable. In Fig. 4a, a snapshot from 2012/04/12 at 05:32 of Stokes drift magnitudes are displayed for the first $9 \mathrm{~m}$ of the three different approximations. In the absence of multidirectional waves, Stokes drift has an exponentially decaying profile. In this figure interestingly, the surface magnitude of the $2 \mathrm{D}_{h}$-SD is half its subsurface value at a depth of $9 \mathbf{~ m}$. This increase of Stokes drift with depth can be attributed to the presence of multiple wave groups since the $1 \mathrm{D}_{h}$ approximations do not exhibit this behavior. Examining the directional distribution for the same time period shown in Fig. 4b, we find that there is sharply peaked swell and diffuse wind seas traveling in approximately opposite directions (roughly east and west respectively). The observations at this time are an extreme example of real-world multidirectional waves.

From examples presented in Section 2, it is clear that both wave spreading and multidirectional waves can affect the magnitude of Stokes drift. To determine if one is more influential, median ratios of the different Stokes drift approximations have been calculated for the two year period and are displayed in Fig. 5 (closed circles). Medians are used for analysis here due to 
large skews and comparisons with mode-centered distributions in Section 4.3; means (open circles) are included for later reference (with Fig. 11). In addition, the shaded interval indicates two-thirds of the distribution centered about the median and is generally not equivalent with the $33 . \overline{3}$ percentile (for a non-normal distribution). The ratios of $1 \mathrm{D}_{h}$-DHH-SD to $1 \mathrm{D}_{h}$-SD have also been displayed for comparison with the prescribed spectra. While it is unclear if the results from prescribed spectra fall within the shaded blue region (due to the use of an $e$-folding depth), the trend is similar with the largest loss at the surface. Since the calculations were limited to depths of $9 \mathrm{~m}$ however, it is also unclear if the observational curve will flatten out as well.

Observational ratios between the $1 \mathrm{D}_{h}$ and $2 \mathrm{D}_{h}$ approximations are illustrated in the other two curves, $2 \mathrm{D}_{h}$-SD to $1 \mathrm{D}_{h}$-SD and $2 \mathrm{D}_{h^{-}}$-SD to $1 \mathrm{D}_{h^{-}}$- $\mathrm{DHH}-$ SD. The (two-third centered) distributions about the median are large in both and are likely caused by multidirectional waves rather than spreading since the distribution for the $1 \mathrm{D}_{h}$-DHH-SD to $1 \mathrm{D}_{h}$-SD ratio is narrowly banded. In Webb and Fox-Kemper (2011), it was identified that $1 \mathrm{D}_{h}$-SD tended to overestimate the $2 \mathrm{D}_{h}$-SD approximation in the model data analyzed by about $33 \%$ (median value) at the surface. This reduction is of a similar magnitude to that found by Ardhuin et al. (2009) and Breivik et al. (2014). While calculated but not shown, the unidirectional overestimation median is nearly double in these observations at $62 \%$. In comparison, the $1 \mathrm{D}_{h^{-}}$ DHH-SD overestimates the $2 \mathrm{D}_{h}$-SD by $28 \%$ at the surface and levels out to about $14 \%$ for depths $3 \mathrm{~m}$ and below. This is still a sizable observational difference but it is a definite improvement. Further discussion on the role of 
wave spreading and multidirectional waves using the OWS-P buoy data will be delayed until Section 7 .

\subsection{Analysis using $1 D_{h}-D H H-S D$ with WAVEWATCH III}

While buoys can provide good spectral data, the data is sparse spatially and it is useful to also compare the results with global model-generated data. A 1-year 2D spectral data set (three-hourly output for the year 2000) has been generated here by WAVEWATCH III (ver. 2.22) and is used to compare differences in Stokes drift approximations.

With the exception of output frequency, model setup is identical with Webb and Fox-Kemper (2011) and implementation details include 25 frequency and 24 directional bins (with an initial and cutoff frequency of $0.0418 \mathrm{~Hz}$ and $0.411 \mathrm{~Hz}$ respectively), $f^{-5}$ tail, and Tolman and Chalikov source terms. In addition, all simulations are forced with CORE2 (Large and Yeager, 2008) winds ${ }^{10}$ with appropriate sea surface temperatures (Hadley SST: Rayner et al., 2006) and sea ice concentrations (Bootstrap Sea Ice Concentrations from Nimbus-7 SMMR and DMSP SSM/I, v2: Comiso, 1999) on a $1^{\circ} \times 1.25^{\circ}$ latitude-longitude grid of $(-78: 78) \times(0: 358.75)$ respectively

In the OWS-P buoy data analyzed in Fig. 5, the majority of uncertainty (in the $1 \mathrm{D}_{h}$ approximations) is likely due to the presence of multidirectional waves. This exercise has been repeated with WAVEWATCH III output and an even larger amount of uncertainty is found on a global scale. ${ }^{11}$ To explore why, scatter plots have been generated in Figs. 6 and 7 to help identify

\footnotetext{
${ }^{10}$ Available from http://data1.gfdl.noaa.gov/nomads/forms/mom4/COREv2.html.

${ }^{11}$ See Fig. 11 for a comparison of mean Stokes drift magnitude ratios (the two-third centered distributions are not shown).
} 
correlations. The dependent values, Stokes drift magnitudes $(\mathrm{m} / \mathrm{s})$ at various depths, are normalized by the $2 \mathrm{D}_{h}$-SD sample maximum (listed in each figure) and shaded by their density distribution. The regions are colored red, green, and blue by the highest $0-30 \%, 31-60 \%$, and $61-90 \%$ centered distributions respectively.

\subsubsection{Surface comparisons}

A comparison of Figs. 6a and 7a, surface magnitudes of $2 \mathrm{D}_{h}$-SD $(y$ axis) versus $1 \mathrm{D}_{h}$-SD and $1 \mathrm{D}_{h}$-DHH-SD ( $x$-axes) respectively, reveals that the sources of error can be cleanly separated. The shaded regions in both figures are approximately equivalent but oriented about different slopes. In Fig. 7a, the upper part of the shaded regions aligns well with the line $y=x$ and indicates that the too shallow slope in Fig. 6a is due to ignoring the effects of wave spreading. The improvement found in slope in Fig. 7a is the direct result from including these effects. It should be pointed out that the alignment in Fig. 7a is not guaranteed. As an example, the slope could have easily been higher than unity if the directional distribution had been too diffuse for higher frequencies. Verifying this agreement is a good test for any potential directional-Stokes-drift-component.

Errors due to multidirectional waves are also present. In both figures, the density distribution normal to the slope of orientation is sharply peaked near the upper part and then gradually descends (in the normal direction with the negative $y$ component). This sharp peak is expected since the ocean surface is rarely a mix of wind seas and swell. In addition, the asymmetry is expected as well. Unlike with wave spreading, the presence of multidirectional waves will only cause an overestimation by the $1 \mathrm{D}_{h}$ approximations and never an 
underestimation.

The correlation holds in Fig. 8 as well, where the surface magnitudes of $1 \mathrm{D}_{h}$-DHH-SD ( $y$-axis) versus $1 \mathrm{D}_{h}$-SD ( $x$-axis) have been plotted for both WAVEWATCH III model and OWS-P buoy data (respectively). In both subfigures, the density distribution normal to the slope of orientation is singularly peaked, which is expected since the magnitudes are invariant to the angle of incidence between the multidirectional waves. In addition, the slopes of the lines the scatter plots fall on are identical (plotted in red with $m=0.795)$ and are in close agreement with the values derived from empirical shapes $(0.799,0.812)$. This implies that reducing the surface $1 \mathrm{D}_{h}$-SD magnitudes by $20 \%$ is a good rule of thumb to approximate directional magnitude losses and correct for wave spreading. This could be very useful since surface magnitudes can be calculated without full knowledge of the $1 \mathrm{D}$ wave spectra (as shown in Webb and Fox-Kemper, 2011). This approach could be extended to include the effects of multidirectional waves globally but it would probably be more effective in a specific region where the statistics of the wave field are known.

\subsubsection{Subsurface comparisons}

Scatter plots of subsurface magnitude ratios for two depths, $3 \mathrm{~m}$ and $9 \mathrm{~m}$, have been generated and are displayed in Figs. 6b,c and 7b,c. As previously, the $1 \mathrm{D}_{h}$-DHH-SD approximation corrects for systematic wave spreading errors while the slope of orientation for the $1 \mathrm{D}_{h}$-SD approximation is less than unity. Interestingly, the slope of the latter increases with the depths displayed and implies that the role of wave spreading diminishes with depth (since the minimum overestimation error decreases). This is consistent with 
prescribed wave spectra results and also suggests that the trend will reverse for deeper depths (albeit for nominal Stokes drift magnitudes).

In both approximations, the density distributions parallel to the slope of orientation become more sharply peaked with depth and can be explained by the higher filtering of wind sea spectra by the exponential component. At the surface, Stokes drift is largely determined by the higher frequencies of the broad-banded wind seas (when multidirectional waves are not significantly present) and this contributes to the broader distribution of magnitudes. However at subsurface depths, the wind sea contribution is quickly attenuated in comparison with the narrow-banded swell. This can be seen in Table 2, where $e$-folding depths of 1 and 3 corresponded to approximately $1 \mathrm{~m}$ and $3 \mathrm{~m}$ for a monochromatic wave with a higher wind sea frequency of $0.34 \mathrm{~Hz}$. In general, the directional magnitude losses due to the interaction of wind seas and swell will decrease with depth (due to the declining role wind seas play) while losses due to the interaction of other multidirectional waves will persist.

\section{Depth-dependent Stokes drift direction}

Up until this point, the discussion has largely focused on Stokes drift magnitude and not direction. Without full knowledge of the 2D wave spectra, it is not immediately clear what the actual direction should be, particularly for subsurface calculations. At the surface, approximations typically assume the direction of Stokes drift is the same as the wind sea and thus the wind direction. But what about subsurface Stokes drift orientation? Should it align 
with the direction of the wind, the mean wave direction, ${ }^{12}$ or a combination of both? Here, the topic of veer is addressed using the same approach as the last section.

In the example in Section 2.2, the direction of the $2 \mathrm{D}_{h}$-SD (for a bichromatic wave with different peak frequencies) varied with depth, with a preference for the directions of the higher and lower frequencies for relatively shallower and deeper depths respectively. This insight is explored further in Figs. 9 and 10 (using scatter plots again) to determine how well the depthdependent and depth-integrated $2 \mathrm{D}_{h}$-SD aligns with the $10 \mathrm{~m}$ surface wind and mean wave directions.

In Figs. 9a and 10a, the $2 \mathrm{D}_{h}$-SD at the surface aligns best with the $10 \mathrm{~m}$ wind. This result is expected since as previously mentioned, the higher frequencies of the wind seas contribute the most to the surface Stokes drift magnitude. However, this correlation no longer holds as depth is increased. As the higher frequencies are filtered by the exponential depth component, the direction of $2 \mathrm{D}_{h}$-SD quickly begins to reorientate toward the mean wave direction. At a depth of $1 \mathrm{~m}$, the correlations for both wind and mean wave directions are already comparable (not shown). By $3 \mathrm{~m}$ and $9 \mathrm{~m}$, the $2 \mathrm{D}_{h^{-}} \mathrm{SD}$ directions are in good and near complete agreement (respectively) with the mean wave direction (Figs. 9b,c and 10b,c).

This veer is consistent with the notion that wind seas dominate surface Stokes drift and swell dominates subsurface Stokes drift. While it is possible to parameterize this transition, the large degree of uncertainty suggests that

\footnotetext{
${ }^{12}$ Following Tolman (2002), the mean wave direction is functionally computed as $\theta_{w}=\operatorname{atan} 2[b, a]$, with $\theta_{w} \in(-\pi, \pi]$ and $(a, b)=\int_{0}^{\infty} \int_{-\pi}^{\pi}(\cos \theta, \sin \theta) \mathcal{S}_{f, \theta}(f, \theta) d \theta d f$.
} 
it might be unwise to do so. If the intermediate subsurface Stokes drift direction is important (as in Sullivan et al., 2012), it is recommended that a spectral wave model is used to calculate this nonlocal, nonlinear vector component.

\section{Depth-integrated Stokes drift magnitude and direction}

Observations of surface slicks (Qazi et al., 2013), computer cards (Weller et al., 1985), high-frequency (HF) radar systems (Chapman et al., 1997), and small drifters (Carrier et al., 2013) tend to emphasize Lagrangian transport of the upper meter of the ocean or less, but the surface Stokes drift is not always the most useful measure of dynamical implications. For one, if the wave field has an $f^{-4}$ spectrum, then the surface $2 \mathrm{D}_{h}$-SD integral does not converge, even though the integral at just $1 \%$ of the peak e-folding depth does (Webb and Fox-Kemper, 2011). As a driving mechanism for Langmuir turbulence, the surface unidirectional Stokes drift may not be as predictive as the depth-integrated form (Harcourt and D'Asaro, 2008; Van Roekel et al., 2012; Sullivan et al., 2012). Furthermore, the depth-integrated Stokes drift depends on a different moment of the wave spectrum than the surface Stokes drift, which lends it very distinct statistics (Myrhaug, 2013b). Thus, in addition to the directional effects of Stokes drift at various depths including the surface, we consider the depth-integrated Stokes drift. ${ }^{13}$

Formally, the Stokes drift approximations $\left(1 \mathrm{D}_{h}-\mathrm{SD}, 1 \mathrm{D}_{h}-\mathrm{DHH}-\mathrm{SD}\right.$, and

\footnotetext{
${ }^{13}$ Sometimes also called the Stokes volume transport.
} 
$2 \mathrm{D}_{h}$-SD) can be depth integrated as

$$
\begin{aligned}
\boldsymbol{u}_{*}^{S \text {-int }} & =\int_{-\infty}^{0} \boldsymbol{u}_{*}^{S}(z) d z \\
& =\int_{-\infty}^{0}\left\{\frac{16 \pi^{3}}{\mathrm{~g}} \int_{0}^{\infty} \boldsymbol{H}_{*}(f) f^{3} S_{f}(f) \exp \left[\frac{8 \pi^{2} f^{2} z}{\mathrm{~g}}\right] d f\right\} d z \\
& =2 \pi \int_{0}^{\infty} \boldsymbol{H}_{*}(f) f S_{f}(f) d f .
\end{aligned}
$$

The reordering of integration is valid here since the integrand is piecewise continuous and non-negative (Roussos, 2013). As previously alluded, we can see that the depth-integrated Stokes drift resembles the first spectral moment (with the addition of a directional component that is dependent upon the approximation used) and that (24) is well-defined for the aforementioned $f^{-4}$ spectrum (since $\left\|\boldsymbol{H}_{*}\right\| \leq 1$ ). The surface Stokes drift depends on the third moment of the spectrum, and thus is significantly more sensitive to high-frequency waves. Here the depth-integrated approximations will use the prefix 'int' (int- $1 \mathrm{D}_{h}$-SD, int-1 $\mathrm{D}_{h}$-DHH-SD, int-2 $\mathrm{D}_{h}$-SD) to distinguish from Stokes drift estimated at a particular depth.

Scatter plots using the depth-integrated Stokes drift approximations are displayed in Figs. 6d, 7d, 9d, and 10d for comparison with previous results. In both magnitude and direction, we can see that the depth-integrated Stokes drift approximations resemble their non-depth-integrated counterparts at subsurface depths, particularly for the case $z=-9 \mathrm{~m}$. Thus, both the depthintegrated and subsurface Stokes drift are aligned with the swell, not wind seas. While these results are intuitively expected, mathematically it is not immediately clear why the resemblance favors a certain depth since there is no $z$-component in (24) to filter out higher frequencies. However, a comparison of the depth-dependent and depth-integrated integrands shows that the 
normalized distributions will be similar as long as the expression,

$$
g(f ; z)=\alpha_{z} f^{2} \exp \left[\frac{8 \pi^{2} f^{2} z}{\mathrm{~g}}\right]
$$

minimizes the filtering of $f \mathcal{S}_{f}$ about an important interval of frequencies for some normalizing constant $\alpha_{z}$. To illustrate, consider a second order Taylor series approximation of (25) centered about $f_{a}$, the median in some frequency subset. Then the approximation will also be centered about $f_{a}$ as long as $g^{\prime}\left(f_{a} ; z\right)=0$, which is equivalent to

$$
z=\mathrm{g} / 8 \pi^{2} f_{a}^{2}
$$

For simplicity, define the subset to contain all of the frequencies in the distributional median bin of $f \mathcal{S}_{f}$. Then solving for and substituting $f_{a}=0.12 \mathrm{~Hz}$ into (26), gives a depth of $8.6 \mathrm{~m}$, with the majority of $f \mathcal{S}_{f}$ attenuated by less than $33 \%$ for the interval covering the subset.

\section{Directional magnitude loss and veer in Stokes drift}

Using three different types of spectra, it has been shown that both wave spreading and multidirectional waves contribute to directional magnitude losses in Stokes drift. Analysis suggests that directional-magnitude-loss errors in the $1 \mathrm{D}_{h}$-SD approximation due to wave spreading are both systematic and removable. Loss errors due to the interaction of locally and remotelygenerated waves however appear to be random and add a large degree of uncertainty in both $1 \mathrm{D}_{h}$ approximations. A detailed quantification of both error types would be useful but only a brief treatment will be given here.

In Fig. 8, surface scatter plot comparisons of $1 \mathrm{D}_{h}$-SD versus $1 \mathrm{D}_{h}$-DHH-SD indicate that the addition of wave spreading does not increase uncertainty 
in the improved $1 \mathrm{D}_{h}$ approximation using either OWS-P buoy or WAVEWATCH III model data. This implies that the physical processes may be independent of each other. In fact, further inspection reveals that the directional effects are largely uncorrelated since the following relation holds to within a percent $(<0.2 \%)$ in both buoy and model data sets:

$$
\begin{aligned}
\left\langle\frac{\left\|\boldsymbol{u}_{2 \mathrm{D}_{h}}^{S}\right\|}{\left\|\boldsymbol{u}_{1 \mathrm{D}_{h}}^{S}\right\|}\right\rangle & =\left\langle\frac{\left\|\boldsymbol{u}_{2 \mathrm{D}_{h}}^{S}\right\|}{\left\|\boldsymbol{u}_{\mathrm{DHH}^{S}}^{S}\right\|} \frac{\left\|\boldsymbol{u}_{\mathrm{DHH}^{S}}^{S}\right\|}{\left\|\boldsymbol{u}_{1 \mathrm{D}_{h}}^{S}\right\|}\right\rangle \\
& \approx\left\langle\frac{\left\|\boldsymbol{u}_{2 \mathrm{D}_{h}}^{S}\right\|}{\left\|\boldsymbol{u}_{\mathrm{DHH}}^{S}\right\|}\right\rangle \times\left\langle\frac{\left\|\boldsymbol{u}_{\mathrm{DHH}}^{S}\right\|}{\left\|\boldsymbol{u}_{1 \mathrm{D}_{h}}^{S}\right\|}\right\rangle .
\end{aligned}
$$

Here, the notation $\langle\cdot\rangle$ is used to denote temporal and unweighted spatial (model only) means. ${ }^{14}$ Since the $2 \mathrm{D}_{h}$-SD to $1 \mathrm{D}_{h}$-DHH-SD and $1 \mathrm{D}_{h}$-DHH$\mathrm{SD}$ to $1 \mathrm{D}_{h}$-SD ratios are largely uncorrelated, both can be compared side by side to measure the directional effects of wave spreading and multidirectional waves separately. In addition, the reciprocal form of $(27)$ also holds $(<0.4 \%)$ and the appropriate means can also be used to assess overestimation errors in the $1 \mathrm{D}_{h}-\mathrm{SD}$ approximation. This additional calculation is necessary since $\langle 1 / X\rangle$ is not necessarily equivalent to $1 /\langle X\rangle$.

To facilitate discussion, mid-Pacific buoy (circles) and global model (squares) mean magnitude ratios have been plotted in Fig. 11 so that the bottom and top halves of the figure can be used to estimate depth-dependent magnitude losses and overestimation errors respectively. In addition, we will refer to the mean $1 \mathrm{D}_{h}$-DHH-SD to $1 \mathrm{D}_{h}$-SD, $2 \mathrm{D}_{h}$-SD to $1 \mathrm{D}_{h}$-DHH-SD, and $2 \mathrm{D}_{h}-\mathrm{SD}$ to $1 \mathrm{D}_{h}-\mathrm{SD}$ ratio curves (and their reciprocals) by the physical processes they represent, namely wave spreading, multidirectional wave(s),

\footnotetext{
${ }^{14}$ No latitudinal adjustments have been made for changing grid cell sizes.
} 
and combined respectively. Due to large skews in the two latter ratio distributions however (see Fig. 5), less emphasis should be placed on the mean values and more on the trends they represent.

At first glance of Fig. 11, we find that the wave spreading curves are coincident between buoy and model data while in contrast the multidirectional wave curves are dissimilar near the surface. The surface differences may be due to the peculiarities of the OWS-P site or model deficiencies (such as errors in long-distance swell propagation or an inability to resolve bimodal wind seas). To illustrate, magnitude scatter plots of the $2 \mathrm{D}_{h}$-SD ( $y$-axis) versus $1 \mathrm{D}_{h^{-}}$-SD and $1 \mathrm{D}_{h}$-DHH-SD ( $x$-axes) have been generated for two different depths $(z=0,-3)$ using OWS-P buoy data in Fig. 12. Notice that higher surface magnitudes slightly deviate from the general patterns observed in WAVEWATCH III model data but do not for subsurface depths. These differences could be caused by bimodal wind seas present in OWS-P buoy data (see Fig. 4b for an example) but not in WAVEWATCH III output. This interesting possibility will be elaborated on in Section 8 .

Examining the bottom half of Fig. 11, we see that there is a 20\% (0.795) reduction in magnitude at the surface due to wave spreading alone and the reduction diminishes somewhat with depth to $14 \%$ at $9 \mathrm{~m}$. In comparison, magnitude losses due to multidirectional wave effects range between $7-23 \%$ at the surface before converging to $17 \%$ for depths $7 \mathrm{~m}$ and deeper. Comparing side by side, wave spreading effects are similar with or dominate multidirectional wave effects from the surface until about a depth of $5 \mathrm{~m}$, where both contribute about a 16\% decrease. For deeper depths, there is better agreement among buoy and model data and multidirectional wave effects 
dominate the magnitude loss. The combined directional magnitude losses range from $26-39 \%$ with a final and subsurface average of $29 \%$.

In the top half of Fig. 11, the multidirectional wave curves are even more dissimilar due to large skew and many of the overestimation errors are larger than what is predicted by their reciprocal counterparts. The overestimation errors due to wave spreading alone are about $26 \%$ at the surface and decrease to $16 \%$ at $9 \mathrm{~m}$. For depths $1 \mathrm{~m}$ and greater, both buoy and model means suggest overestimation errors due to multidirectional waves will dominate, ranging from an additional 26-43\%. The combined overestimation errors are particularly substantial and range from approximately $45-71 \%$ (both are at the surface).

As a companion comparison, mean ratios have been tabulated for the depth-integrated approximations in Table 4 using WAVEWATCH III model data. Magnitude losses from wave spreading and multidirectional wave effects alone are $16 \%$ and $17 \%$ respectively, giving a combined loss of $30 \%$. Interestingly, overestimation errors from multidirectional wave effects are more than double than those of wave spreading at $41 \%$ and $19 \%$ respectively, giving a combined error of $68 \%$. These results are similar with depth-dependent Stokes drift subsurface ratios, which in turn is consistent with previous analysis in Section 6.

In addition to magnitude, the direction of Stokes drift is also affected and multidirectional waves will generate a directional veer. Instead of examining mean ratios however, a rough estimate of global directional errors are made using confidence intervals from scatter plots. For surface spectral moment approximations, a typical $10 \mathrm{~m}$ wind alignment assumption will lead to a 
random directional error. Examining Fig. 9a, we find that the 30/60/90\% confidence intervals are bounded (approximately) by $\pm 0.12 / 0.28 / 0.84$ radians $( \pm 7 / 16 / 48 \mathrm{deg})$. Contrast this with Fig. $9 \mathrm{c}$ where at a $9 \mathrm{~m}$ depth, the $30 / 60 / 90 \%$ confidence interval bounds using a mean wave direction assumption are only $\pm 0.05 / 0.09 / 0.21$ radians $( \pm 3 / 5 / 12 \mathrm{deg})$. Likewise for the depth-integrated form in Fig. 9d, the 30/60/90\% confidence interval bounds for the same assumption are $\pm 0.09 / 0.16 / 0.35$ radians $( \pm 5 / 9 / 20 \mathrm{deg})$.

\section{Discussion}

Directional spreading is an active field of research and there have been many advances since the classical measurements of Mitsuyasu et al. (1975), Hasselmann et al. (1980), and Donelan et al. (1985). There are now a variety of techniques (Fourier Expansion Method, Maximum Entropy Method, Maximum Likelihood Method, and Wavelet Directional Method) to extract the directional spectra and accuracy will depend upon the method chosen (Young, 1994; Alves and Melo, 1999; Young, 2010). In addition, there is now strong evidence that fetch-limited waves in deep water are bimodal for frequencies higher than the peak frequency (Young et al., 1995; Ewans, 1998; Alves and Melo, 1999; Hwang et al., 2000). While not demonstrated here, a bimodal directional distribution will generate multidirectional magnitude losses (in addition to wave spreading ones) that are not present in a unimodal directional distribution. This could be a particularly important result for any model simulation since the $2 \mathrm{D}_{h}$-SD approximation will likely overestimate Stokes drift if the bimodal wind seas are not resolved properly.

As is evident from analysis, near surface Stokes drift magnitudes are 
particularly sensitive to the higher-frequency wind seas. This implies that any improved $1 \mathrm{D}_{h}$-SD estimate will also be sensitive to wind seas near the surface and any wave spreading function that is too broad (or narrow) will overestimate (or underestimate) magnitude losses. At this early stage of investigation, no attempt has been made to measure the directional spreading in the OWS-P buoy or WAVEWATCH III model data. This endeavor certainly would be useful in future studies for measuring the effectiveness of the spreading function chosen (particularly for mixed seas conditions) and conducting any sensitivity analyses of the improved estimate (particularly near the surface). In addition, the measured directional spreading might also be a useful metric for comparing $2 \mathrm{D}_{h}$-SD calculations from different data sets.

One of the shortcomings of this study is that the spreading functions considered here are designed for fetch-limited conditions and are likely to be inaccurate at measuring swell (Alves and Melo, 1999). In addition, these classical spreading functions are only tuned for one wave group. As a result, the directional spread for wind seas is likely to be too broad when large swell are also present (i.e., the peak frequency from swell is used instead to determine the spread for wind seas) (Alves and Melo, 1999). This is the main reason why the DHH-B directional-Stokes-drift-component was not used in this analysis. Most other spreading functions, including DHH-B, broaden sharply for large values of $f / f_{p}$. However, the DHH spreading function (and more importantly the DHH directional-Stokes-drift-component) is constant for $f / f_{p}>1.6$ (see Fig. 2) and minimizes the risk of underestimating Stokes drift when both swell and wind seas are present.

Regardless though of the wave spreading function chosen for the direc- 
tional-Stokes-drift-component, the directional effects on magnitude (due to wave spreading and multidirectional waves) should remain largely uncorrelated. This is due to the similarities of the wave spreading functions and on the mechanics involved. However, spreading losses associated with each improved $1 \mathrm{D}_{h}$ estimate should vary. Studies by Young (1994) have shown the Mitsuyasu et al. (1975) and Hasselmann et al. (1980) distributions are artificially broad; as a result they are likely to overestimate the magnitude losses. In addition, studies by Donelan et al. (1985), Young and Van Vledder (1993), and Young (1994) have questioned their dependence on wave age. As discussed in Sec. 4.3.1, agreement between any improved $1 \mathrm{D}_{h}$-SD estimate and $2 \mathrm{D}_{h}$-SD for wind seas is not guaranteed and fact that $1 \mathrm{D}_{h}$-DHH-SD is in agreement (aside from OWS-P buoy data near the surface), should not be overlooked. Based on the reasons discussed here, the DHH wave spreading function appears to be an adequate choice for this exploratory study.

\subsection{Thoughts on improving $1 D$ spectral calculations of Stokes drift}

Fully reconciling unidirectional and multidirectional approximations is a difficult task and this work has been a first step at identifying and quantifying differences in magnitude and direction. While the $1 \mathrm{D}_{h}$-DHH-SD and int-1 $\mathrm{D}_{h}$-DHH-SD approximations have been used here as a proxy to examine these differences, they certainly can be used to calculate depth-dependent and depth-integrated magnitudes when 2D spectra are not available. However as shown, both approximations will not include the effects of multiple wave groups and these additional magnitude losses will need to be estimated (approximately $7-23 \%$ and 17\% respectively in the data examined here).

Unfortunately, predicting the depth-dependent Stokes drift direction is 
less straightforward and as shown, there can even be large differences at the surface with the commonly assumed (wind) direction. However, it may be possible to parametrize the Stokes drift veer from (approximate) alignment with wind at the surface to the mean wave direction at deeper depths using a method similar to Section 6. As also noted by Breivik et al. (2014), direction is less of an issue with the depth-integrated Stokes drift since it will mostly align with the mean wave direction.

The best approach however for improving 1D spectral calculations would likely involve spectral partitioning. In theory, this could remove errors both in magnitude and direction and would not require any prior knowledge or assumptions about the sea state. If the 1D frequency spectra were partitioned into swell and wind seas as $\mathcal{S}_{f}=\mathcal{S}_{\text {swell, } f}+\mathcal{S}_{\text {wind }, f}$ (such as using the method outlined in Portilla et al. (2009)), then (22) could be rewritten as

$$
\begin{aligned}
\boldsymbol{u}_{\mathrm{Par}}^{S}= & \frac{16 \pi^{3}}{\mathrm{~g}}\left\{\hat{\boldsymbol{e}}^{\mathrm{swell}} \int_{0}^{\infty} H_{\mathrm{DHH}}\left(f / f_{p}\right) f^{3} S_{\text {swell }, f}(f) \exp \left[\frac{8 \pi^{2} f^{2} z}{\mathrm{~g}}\right] d f\right. \\
& \left.+\hat{\boldsymbol{e}}^{\text {wind }} \int_{0}^{\infty} H_{\mathrm{DHH}}\left(f / f_{p}\right) f^{3} S_{\text {wind }, f}(f) \exp \left[\frac{8 \pi^{2} f^{2} z}{\mathrm{~g}}\right] d f\right\}
\end{aligned}
$$

where $\hat{\boldsymbol{e}}^{\text {swell }}$ and $\hat{\boldsymbol{e}}^{\text {wind }}$ are simply the mean wave and wind directions. ${ }^{15}$ Notice that this formulation would remove the directionally-diffuse-wind-seas problem when large swell are present. In addition, it would also allow the use of different directional-Stokes-drift-components $\left(H_{*}\right)$ that could be tailored (and simplified) for fully-developed conditions or bimodal wind seas. While computation would certainly increase, it would likely provide the best 1D spectral estimate of the multidirectional Stokes drift for random seas and

\footnotetext{
${ }^{15}$ This approach could also be used with (24), the depth-integrated form.
} 
warrants further investigation.

\section{Summary}

Here, the directional effects of wave spreading and multidirectional waves on Stokes drift have been explored in the random sea approximations. Since the unidirectional $1 \mathrm{D}_{h}$-SD ignores these features, a new $1 \mathrm{D}_{h}$-DHH-SD approximation is defined to incorporate the systematic effects of wave spreading. This approximation is based on observational studies of Donelan et al. (1985) and uses a Padé approximate to simplify the solution. Both 1D frequency spectra approximations are compared with the $2 \mathrm{D}_{h}$-SD approximation using prescribed, observational, and model-generated wave spectra and it is shown that the improved $1 \mathrm{D}_{h}$-DHH-SD reduces magnitude errors in overestimation. In addition, the directional magnitude losses due to wave spreading and multidirectional waves are largely uncorrelated and affect both the magnitude and direction of Stokes drift in a nonlinear fashion that is sensitive with depth. Furthermore, directional effects in depth-integrated approximations are also considered and results are consistent with depth-dependent subsurface ones.

\section{Acknowledgements}

A portion of this work first appeared in the doctoral dissertation of Webb (2013). A. Webb and B. Fox-Kemper were partially supported by NASA ROSES-NNX09AF38G and NSF OCE-0934737. B. Fox-Kemper was also partially supported by NSF OCE-1258907. B. Fox-Kemper would like to 
thank the Kavli Institute for Theoretical Physics and its Wave-Flow Interaction workshop for additional support during the completion of this manuscript (preprint NSF-KITP-14-084, supported by NSF PHY11-25915). Assistance from Jim Thomson and Ramsey Harcourt with obtaining and interpreting the OWS-P buoy data is greatly appreciated. Moreover, MEM Matlab codes written by Paul Jessen (edited by Jim Thomson) and Tyson Hilmer proved invaluable in constructing the directional-wave-information observational dataset. And finally, the authors would like to thank three anonymous reviewers for their careful assessment and comments which significantly improved this manuscript. 
Alves, J., Melo, E., 1999. On the measurement of directional wave spectra at the southern Brazilian coast. Applied Ocean Research 21, 295-309.

Ardhuin, F., Marié, L., Rascle, N., Forget, P., Roland, A., 2009. Observation and estimation of Lagrangian, Stokes, and Eulerian currents induced by wind and waves at the sea surface. Journal of Physical Oceanography 39, $2820-2838$.

Babanin, A.V., Haus, B.K., 2009. On the existence of water turbulence induced by nonbreaking surface waves. Journal of Physical Oceanography $39,2675-2679$.

Banner, M.L., 1990. Equilibrium spectra of wind-waves. Journal of Physical Oceanography 20, 966-984.

Belcher, S.E., Grant, A.A.L.M., Hanley, K.E., Fox-Kemper, B., Van Roekel, L., Sullivan, P.P., Large, W.G., Brown, A., Hines, A., Calvert, D., Rutgersson, A., Petterson, H., Bidlot, J., Janssen, P.A.E.M., Polton, J.A., 2012. A global perspective on Langmuir turbulence in the ocean surface boundary layer. Geophysical Research Letters 39, L18605, 9pp. URL: http: //dx.doi.org/10.1029/2012GL052932, doi:10.1029/2012GL052932.

Belka, D., Schwendeman, M., Thomson, J., Cronin, M., 2014. Historical Wave and Wind Observations at Ocean Station P. Technical Report APL-UW 1407. Applied Physics Laboratory, University of Washington. Seattle, WA. URL: http://www.apl.washington.edu/research/ downloads/publications/tr_1407.pdf. 
Bender, C.M., Orszag, S.A., 1978. Advanced Mathematical Methods for Scientists and Engineers I: Asymptotic Methods and Perturbation Theory. McGraw-Hill, New York, NY.

Breivik, Ø., Janssen, P.A.E.M., Bidlot, J.R., 2014. Approximate Stokes drift profiles in deep water. Journal of Physical Oceanography 44, 2433-2445. doi:10.1175/JPO-D-14-0020.1.

Carrasco, A., Semedo, A., Isachsen, P.E., Christensen, K.H., Saetra, Ø., 2014. Global surface wave drift climate from ERA-40: The contributions from wind-sea and swell. Ocean Dynamics 64, 1815-1829.

Carrier, M.J., Ngodock, H., Smith, S., Jacobs, G., Muscarella, P., Ozgokmen, T., Haus, B., Lipphardt, B., 2013. Impact of assimilating ocean velocity observations inferred from Lagrangian drifter data using the NCOM-4DVAR. Monthly Weather Review 142, 1509-1524.

Cavaleri, L., Fox-Kemper, B., Hemer, M., 2012. Wind waves in the coupled climate system. Bulletin of the American Meteorological Society 93, 16511661. URL: http://dx .doi .org/10.1175/BAMS-D-11-00170.1.

Chapman, R., Shay, L., Graber, H., Edson, J., Karachintsev, A., Trump, C., Ross, D., 1997. On the accuracy of HF radar surface current measurements: Intercomparisons with ship-based sensors. Journal of Geophysical Research: Oceans (1978-2012) 102, 18737-18748.

Comiso, J., 1999. Bootstrap sea ice concentrations for NIMBUS-7 SMMR and DMSP SSM/I. Technical Report 2. National Snow and Ice Data Cen- 
ter. Boulder, CO. URL: http://nsidc.org/data/nsidc-0079.html. Updated 2008 .

Craik, A.D.D., Leibovich, S., 1976. Rational model for Langmuir circulations. Journal of Fluid Mechanics 73, 401-426.

Dalrymple, R.A., Dean, R.G., 1984. Water Wave Mechanics for Engineers and Scientists. Volume 2. Prentice-Hall, Singapore.

D’Asaro, E., Thomson, J., Shcherbina, A., Harcourt, R., Cronin, M., Hemer, M., Fox-Kemper, B., 2014. Quantifying upper ocean turbulence driven by surface waves. Geophysical Research Letters 41, 102-107.

D'Asaro, E.A., 2014. Turbulence in the upper-ocean mixed layer. Annual Review of Marine Science 6, 101-115.

Donelan, M.A., Hamilton, J., Hui, W.H., 1985. Directional spectra of windgenerated waves. Philosophical Transactions of the Royal Society of London Series A-Mathematical Physical and Engineering Sciences 315, 509562 .

Ewans, K.C., 1998. Observations of the directional spectrum of fetch-limited waves. Journal of Physical Oceanography 28, 495-512.

Fan, Y., Griffies, S.M., 2014. Impacts of parameterized Langmuir turbulence and non-breaking wave mixing in global climate simulations. Journal of Climate 27, 4752-4775.

Forristall, G.Z., Ewans, K.C., 1998. Worldwide measurements of directional 
wave spreading. Journal of Atmospheric and Oceanic Technology 15, 440469.

Gommenginger, C.P., Srokosz, M.A., Challenor, P.G., Cotton, P.D., 2003. Measuring ocean wave period with satellite altimeters: A simple empirical model. Geophysical Research Letters 30. doi:DOI10.1029/2003GL017743.

Grant, A.L.M., Belcher, S.E., 2009. Characteristics of Langmuir turbulence in the ocean mixed layer. Journal of Physical Oceanography 39, 1871-1887. doi:DOI10.1175/2009JP04119.1.

Harcourt, R.R., 2013. A second-moment closure model of Langmuir turbulence. Journal of Physical Oceanography 43, 673-697.

Harcourt, R.R., D’Asaro, E.A., 2008. Large-eddy simulation of Langmuir turbulence in pure wind seas. Journal of Physical Oceanography 38, 15421562. doi:DOI10.1175/2007 JP03842.1.

Hasselmann, D.E., Dunckel, M., Ewing, J.A., 1980. Directional wave spectra observed during JONSWAP 1973. Journal of Physical Oceanography 10, $1264-1280$.

Hasselmann, K., Ross, D.B., Muller, P., Sell, W., 1976. Parametric wave prediction model. Journal of Physical Oceanography 6, 200-228.

Holthuijsen, L.H., 2007. Waves in Oceanic and Coastal Waters. Cambridge University Press, New York, NY. URL: http://www.loc.gov/catdir/ toc/fy0711/2007274988.html. 
Huang, C.J., Qiao, F., Song, Z., Ezer, T., 2011. Improving simulations of the upper ocean by inclusion of surface waves in the Mellor-Yamada turbulence scheme. Journal of Geophysical Research-Oceans 116, C01007. doi:DOI10.1029/2010JC006320.

Hwang, P.A., Wang, D.W., Walsh, E.J., Krabill, W.B., Swift, R.N., 2000. Airborne measurements of the wavenumber spectra of ocean surface waves. Part II: Directional distribution*. Journal of Physical Oceanography 30, $2768-2787$.

Kantha, L.H., Clayson, C.A., 2004. On the effect of surface gravity waves on mixing in the oceanic mixed layer. Ocean Modelling 6, 101-124. doi:DOI10. 1016/S1463-5003(02)00062-8.

Kenyon, K.E., 1969. Stokes drift for random gravity waves. Journal of Geophysical Research 74, 6991-6994.

Large, W.G., Yeager, S.G., 2008. The global climatology of an interannually varying air-sea flux data set. Climate Dynamics 33, 341-364. doi:DOI10. 1007/s00382-008-0441-3.

Liu, G., Perrie, W.A., He, Y., 2014. Ocean surface Stokes drift from scatterometer observations. International Journal of Remote Sensing 35, 19661978.

Luke, Y.L., 1969. The Special Functions and Their Approximations. Academic Press, New York, NY. 
Lygre, A., Krogstad, H.E., 1986. Maximum entropy estimation of the directional distribution in ocean wave spectra. Journal of Physical Oceanography 16, 2052-2060.

McWilliams, J.C., Fox-Kemper, B., 2013. Oceanic wave-balanced surface fronts and filaments. Journal of Fluid Mechanics 730, 464-490. URL: http: //dx.doi.org/10.1017/jfm.2013.348, doi:10.1017/jfm.2013.348.

McWilliams, J.C., Huckle, E., Liang, J.H., Sullivan, P.P., 2012. The wavy Ekman layer: Langmuir circulations, breaking waves, and Reynolds stress. Journal of Physical Oceanography 42, 1793-1816.

McWilliams, J.C., Restrepo, J.M., 1999. The wave-driven ocean circulation. Journal of Physical Oceanography 29, 2523-2540.

Mitsuyasu, H., Tasai, F., Suhara, T., Mizuno, S., Ohkusu, M., Honda, T., Rikiishi, K., 1975. Observations of the directional spectrum of ocean waves using a cloverleaf buoy. Journal of Physical Oceanography 5, 750-760.

Myrhaug, D., 2013a. Some statistical aspects of wave-induced drift in sea states. Coastal Engineering 78, 53-56.

Myrhaug, D., 2013b. Stokes drift estimation based on long-term variation of wave conditions. Proceedings of the Institution of Mechanical Engineers, Part M: Journal of Engineering for the Maritime Environment 1475090213506699 .

Myrhaug, D., Wang, H., Holmedal, L.E., 2014. Stokes drift estimation for deep water waves based on short-term variation of wave conditions. Coastal Engineering 88, 27-32. 
O'Reilly, W.C., Herbers, T.H.C., Seymour, R.J., Guza, R.T., 1996. A comparison of directional buoy and fixed platform measurements of Pacific swell. Journal of Atmospheric and Oceanic Technology 13, 231-238.

Pierson, Jr., W.J., Moskowitz, L., 1964. A proposed spectral form for fully developed wind seas based on the similarity theory of S. A. Kitaigorodskii. Journal of Geophysical Research 69, 5181-5190.

Portilla, J., Ocampo-Torres, F.J., Monbaliu, J., 2009. Spectral partitioning and identification of wind sea and swell. Journal of Atmospheric and Oceanic Technology 26, 107-122.

Qazi, W.A., Emery, W.J., Fox-Kemper, B., 2013. Computing ocean surface currents over the coastal California Current System using 30-minute lag sequential SAR images. IEEE Transactions on Geoscience and Remote Sensing 52, 7559-7580.

Rascle, N., Ardhuin, F., 2013. A global wave parameter database for geophysical applications. Part 2: Model validation with improved source term parameterization. Ocean Modelling 70, 174-188.

Rayner, N.A., Brohan, P., Parker, D.E., Folland, C.K., Kennedy, J.J., Vanicek, M., Ansell, T.J., Tett, S.F.B., 2006. Improved analyses of changes and uncertainties in sea surface temperature measured in situ since the mid-nineteenth century: The HadSST2 dataset. Journal of Climate 19, 446-469.

Röhrs, J., Christensen, K.H., Vikebø, F., Sundby, S., Saetra, Ø., Broström, 
G., 2014. Wave-induced transport and vertical mixing of pelagic eggs and larvae. Limnology and Oceanography 59, 1213-1227.

Roussos, I.M., 2013. Improper Riemann Integrals. CRC Press, Boca Raton, FL.

Sullivan, P.P., McWilliams, J.C., 2010. Dynamics of winds and currents coupled to surface waves. Annual Review of Fluid Mechanics 42, 19-42. doi:DOI10.1146/annurev-fluid-121108-145541.

Sullivan, P.P., Romero, L., McWilliams, J.C., Melville, W.K., 2012. Transient evolution of Langmuir turbulence in ocean boundary layers driven by hurricane winds and waves. Journal of Physical Oceanography 42, 19591980.

Thomson, J., D’Asaro, E., Cronin, M., Rogers, W., Harcourt, R., Shcherbina, A., 2013. Waves and the equilibrium range at Ocean Weather Station P. Journal of Geophysical Research: Oceans 118, 5951-5962.

Thorpe, S., 1985. Small-scale processes in the upper ocean boundary layer. Nature 318, 519-522.

Tolman, H.L., 2002. User manual and system documentation of WAVEWATCH-III version 2.22. Technical Report 222. NOAA / NWS / NCEP / MMAB.

Van Roekel, L.P., Fox-Kemper, B., Sullivan, P.P., Hamlington, P.E., Haney, S.R., 2012. The form and orientation of Langmuir cells for misaligned winds and waves. Journal of Geophysical Research: Oceans 117, C05001, 
22pp. URL: http://dx.doi.org/10.1029/2011JC007516, doi:10.1029/ $2011 \mathrm{JC} 007516$.

Webb, A., 2013. Stokes Drift and Meshless Wave Modeling. Ph.D. thesis. University of Colorado Boulder.

Webb, A., Fox-Kemper, B., 2011. Wave spectral moments and Stokes drift estimation. Ocean Modelling 40, 273-288.

Weller, R.A., Dean, J.P., Price, J.F., Francis, E.A., Marra, J., Boardman, D.C., 1985. Three-dimensional flow in the upper ocean. Science 227, 15521556.

Young, I., 1994. On the measurement of directional wave spectra. Applied Ocean Research 16, 283-294.

Young, I., 2010. The form of the asymptotic depth-limited wind-wave spectrum: Part III-Directional spreading. Coastal Engineering 57, 30-40.

Young, I., Van Vledder, G.P., 1993. A review of the central role of nonlinear interactions in wind-wave evolution. Philosophical Transactions of the Royal Society of London. Series A: Physical and Engineering Sciences 342, $505-524$.

Young, I., Verhagen, L., Banner, M., 1995. A note on the bimodal directional spreading of fetch-limited wind waves. Journal of Geophysical Research: Oceans (1978-2012) 100, 773-778. 


\section{Appendix A. The DHH-B directional-Stokes-drift-component}

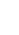
ing function was not constant for $f / f_{p} \geq 1.6$ and proposed the following modification (Ewans, 1998):

$$
\beta_{\mathrm{B}}\left(f / f_{p}\right)=10^{-0.4} \exp \left[0.8393 \ln [10.00]\left(f / f_{p}\right)^{-1.134}\right], \quad f / f_{p} \geq 1.6
$$

Following Section 3.4, a directional-Stokes-drift-component was approximated (using a Padé approximate of order [2/2]) for the Banner-modified directional distribution (termed $D H H-B$ here) as

$$
\boldsymbol{H}_{\mathrm{DHH}-\mathrm{B}}\left(f ; f_{p}, \bar{\theta}\right)=(\cos \bar{\theta}, \sin \bar{\theta}, 0) \frac{0.5 f_{p}^{2}+0.22 f_{p} f+0.19 f^{2}}{f_{p}^{2}-0.81 f_{p} f+0.79 f^{2}}, \quad f / f_{p} \geq 1.6
$$

The new directional-Stokes-drift-component approximation has a maximum relative error of $7 \times 10^{-5}$.

\section{Appendix B. Stokes drift spectral tail calculations}

4 ciest integrating cutoff frequency $f_{\tilde{c}}$ is introduced for flexibility in discretization. ${ }^{16}$ For some positive $p$ and $f_{c}$, let the spectral tail be governed by

$$
\mathcal{S}_{f \theta}(f, \theta)=\left(\frac{f_{c}}{f}\right)^{p} \mathcal{S}_{f \theta}\left(f_{c}, \theta\right), \quad f \geq f_{\tilde{c}} \geq f_{c} .
$$

\footnotetext{
${ }^{16}$ For practical purposes, $f_{\tilde{c}}$ can be viewed as equivalent to $f_{c}$ here.
} 
${ }_{959}$ Then the depth-dependent and depth-integrated $2 \mathrm{D}_{h}$-SD tail contributions 960 for $f \geq f_{\tilde{c}}$ are

$$
\begin{aligned}
\boldsymbol{u}_{2 \mathrm{D}, \text { tail }}^{S}= & \frac{16 \pi^{3}}{\mathrm{~g}} f_{c}^{p} \int_{-\pi}^{\pi}(\cos \theta, \sin \theta, 0) \mathcal{S}_{f \theta}\left(f_{c}, \theta\right) \\
& \times\left\{\int_{f_{\tilde{c}}}^{\infty} f^{3-p} \exp \left[-\frac{8 \pi^{2}|z|}{\mathrm{g}} f^{2}\right] d f\right\} d \theta
\end{aligned}
$$

961 and

$$
\boldsymbol{u}_{2 \mathrm{D}, \text { tail }}^{S \text {-int }}=2 \pi f_{c}^{p} \int_{-\pi}^{\pi}(\cos \theta, \sin \theta, 0) \mathcal{S}_{f \theta}\left(f_{c}, \theta\right)\left\{\int_{f_{\tilde{c}}}^{\infty} f^{1-p} d f\right\} d \theta .
$$

962 Appendix B.1. Subsurface Stokes drift tail

${ }_{963}$ Note that for $\alpha, f_{\tilde{c}}>0(\alpha \in \mathbb{R})$,

$$
\begin{aligned}
I=\int_{f_{\tilde{c}}}^{\infty} f^{3-p} \exp \left[-\alpha f^{2}\right] d f & =\frac{\alpha^{\frac{p}{2}-2}}{2} \int_{\alpha f_{\tilde{c}}^{2}}^{\infty} t^{2-\frac{p}{2}} \exp [-t] d t \\
& =\frac{\alpha^{\frac{p}{2}-2}}{2} \Gamma\left[2-\frac{p}{2}, \alpha f_{\tilde{c}}^{2}\right],
\end{aligned}
$$

964 where $\Gamma[s, x]$ is the upper incomplete gamma function. Then for $\alpha=$ $9658 \pi^{2}|z| / \mathrm{g}$,

$$
\begin{aligned}
\left.\boldsymbol{u}_{2 \mathrm{D}, \text { tail }}^{S}\right|_{z \neq 0}= & \frac{8 \pi^{3}}{\mathrm{~g}} \alpha^{\frac{p}{2}-2} f_{c}^{p} \Gamma\left[2-\frac{p}{2}, \alpha f_{\tilde{c}}^{2}\right] \\
& \times \int_{-\pi}^{\pi}(\cos \theta, \sin \theta, 0) \mathcal{S}_{f \theta}\left(f_{c}, \theta\right) d \theta .
\end{aligned}
$$


Three common values of $p$ are $4,4.5$, and 5 . These reduce to

$$
\begin{aligned}
\left.\boldsymbol{u}_{2 \mathrm{D}, \text { tail }, p=4}^{S}\right|_{z \neq 0}= & \frac{8 \pi^{3}}{\mathrm{~g}} f_{c}^{4} \Gamma\left[0, \alpha f_{\tilde{c}}^{2}\right] \int_{-\pi}^{\pi}(\cos \theta, \sin \theta, 0) \mathcal{S}_{f \theta}\left(f_{c}, \theta\right) d \theta \\
\left.\boldsymbol{u}_{2 \mathrm{D}, \text { tail }, p=4.5}^{S}\right|_{z \neq 0}= & \frac{8 \pi^{3}}{\mathrm{~g}} \alpha^{\frac{1}{4}} f_{c}^{\frac{9}{2}} \Gamma\left[-\frac{1}{4}, \alpha f_{\tilde{c}}^{2}\right] \\
& \times \int_{-\pi}^{\pi}(\cos \theta, \sin \theta, 0) \mathcal{S}_{f \theta}\left(f_{c}, \theta\right) d \theta \\
\left.\boldsymbol{u}_{2 \mathrm{D}, \text { tail }, p=5}^{S}\right|_{z \neq 0}= & \frac{8 \pi^{3}}{\mathrm{~g}} \alpha^{\frac{1}{2}} f_{c}^{5} \Gamma\left[-\frac{1}{2}, \alpha f_{\tilde{c}}^{2}\right] \int_{-\pi}^{\pi}(\cos \theta, \sin \theta, 0) \mathcal{S}_{f \theta}\left(f_{c}, \theta\right) d \theta \\
= & \frac{16 \pi^{3}}{\mathrm{~g}} f_{c}^{4}\left[\exp \left[-\alpha f_{\tilde{c}}^{2}\right]-\sqrt{\pi \alpha f_{\tilde{c}}^{2}}\left(1-\operatorname{erf}\left[\sqrt{\alpha f_{\tilde{c}}^{2}}\right]\right)\right] \\
& \times \int_{-\pi}^{\pi}(\cos \theta, \sin \theta, 0) \mathcal{S}_{f \theta}\left(f_{c}, \theta\right) d \theta
\end{aligned}
$$

967

where the error function (erf : $\left.\mathbb{R}_{+}^{*} \rightarrow[0,1]\right)$ is defined as

$$
\operatorname{erf}[x]=\frac{2}{\sqrt{\pi}} \int_{0}^{x} \exp \left[-t^{2}\right] d t
$$

Appendix B.2. Surface Stokes drift tail

If $z=0$, then the Stokes drift tail is restricted to $p>4$ and simplifies to

$$
\left.\boldsymbol{u}_{2 \mathrm{D}, \text { tail }, p>4}^{S}\right|_{z=0}=\frac{16 \pi^{3} f_{\tilde{c}}^{4}}{\mathrm{~g}(p-4)}\left(\frac{f_{c}}{f_{\tilde{c}}}\right)^{p} \int_{-\pi}^{\pi}(\cos \theta, \sin \theta, 0) \mathcal{S}_{f \theta}\left(f_{c}, \theta\right) d \theta .
$$

\section{Appendix B.3. Depth-integrated Stokes drift tail}

Similarly for $p>2$, the tail contribution to the depth-integrated Stokes drift is

$$
\boldsymbol{u}_{2 \mathrm{D}, \mathrm{tail}, p>2}^{S-\mathrm{int}}=\frac{2 \pi f_{\tilde{c}}^{2}}{p-2}\left(\frac{f_{c}}{f_{\tilde{c}}}\right)^{p} \int_{-\pi}^{\pi}(\cos \theta, \sin \theta, 0) \mathcal{S}_{f \theta}\left(f_{c}, \theta\right) d \theta .
$$

The reader may note that a similar form to some of these spectral tail equations have previously appeared (Webb, 2013; Breivik et al., 2014). 


\section{Appendix C. Numerical Stokes drift calculations}

Discretization of Stokes drift for model inclusion is outlined in this section. Let the number of spectral bins, centers, and widths be represented by $N_{*}, *_{i}, \Delta *_{i}$ respectively. In addition, all spectral tail and depth-dependent subsurface calculations use $p=5$ and $\alpha=8 \pi^{2}|z| / g$ respectively. There are two natural choices here for the integrating cutoff frequency $f_{\tilde{c}}$. The first choice, $f_{\tilde{c}}:=f_{c}+\Delta f_{N_{f}} / 2$, does not require any modification to the discretizations presented. The second choice, $f_{\tilde{c}}:=f_{c}$, uses only half of the last frequency bin (i.e., $f_{N_{f}}-\Delta f_{N_{f}} / 2 \leq f \leq f_{N_{f}}$ ) for all numerical calculations. This latter method was employed in this manuscript.

Appendix C.1. 2D $D_{h}-S D$

The subsurface and surface $2 \mathrm{D}_{h}$-SD numerical approximations are

$$
\begin{aligned}
\boldsymbol{u}_{2 \mathrm{D}}^{S} \approx & \frac{2 \pi \alpha}{|z|} \sum_{j=1}^{N_{\theta}} \Delta \theta_{j}\left(\cos \theta_{j}, \sin \theta_{j}, 0\right)\left\{\left[\sum_{i=1}^{N_{f}} \Delta f_{i} f_{i}^{3} \exp \left[-\alpha f_{i}^{2}\right] S_{f \theta}\left(f_{i}, \theta_{j}\right)\right]\right. \\
& \left.+f_{c}^{4}\left[\exp \left[-\alpha f_{\tilde{c}}^{2}\right]-\sqrt{\pi \alpha f_{\tilde{c}}^{2}}\left(1-\operatorname{erf}\left[\sqrt{\alpha f_{\tilde{c}}^{2}}\right]\right)\right] \mathcal{S}_{f \theta}\left(f_{c}, \theta_{j}\right)\right\}, \\
\left.\boldsymbol{u}_{2 \mathrm{D}}^{S}\right|_{z=0} \approx & \frac{16 \pi^{3}}{\mathrm{~g}} \sum_{j=1}^{N_{\theta}} \Delta \theta_{j}\left(\cos \theta_{j}, \sin \theta_{j}, 0\right) \\
& \times\left\{\left[\sum_{i=1}^{N_{f}} \Delta f_{i} f_{i}^{3} S_{f \theta}\left(f_{i}, \theta_{j}\right)\right]+\frac{f_{c}^{5}}{f_{\tilde{c}}} \mathcal{S}_{f \theta}\left(f_{c}, \theta_{j}\right)\right\} .
\end{aligned}
$$


Appendix C.2. Directional $1 D_{h}-S D$

Recall that for a generic $\|\boldsymbol{H}\| \neq 1$, the directional $1 \mathrm{D}_{h}$-SD can be written as

$$
\boldsymbol{u}_{1 \mathrm{D}-\mathrm{dir}}^{S}=\hat{\boldsymbol{e}}^{\mathrm{w}} \frac{16 \pi^{3}}{g} \int_{0}^{\infty} H(f) f^{3} S_{f}(f) \exp \left[\frac{8 \pi^{2} f^{2}}{g} z\right] d f,
$$

where $\hat{\boldsymbol{e}}^{\mathrm{w}}$ is the assumed direction and $H(f)=\|\boldsymbol{H}(f)\|$ is the directionalStokes-drift-component magnitude. The resultant subsurface and surface directional $1 \mathrm{D}_{h}$-SD numerical approximations are

$$
\begin{gathered}
\left\|\boldsymbol{u}_{1 \mathrm{D}-\text { dir }}^{S}\right\| \approx \frac{2 \pi \alpha}{|z|}\left\{\left[\sum_{i=1}^{N_{f}} \Delta f_{i} H\left(f_{i}\right) f_{i}^{3} \exp \left[-\alpha f_{i}^{2}\right] S_{f}\left(f_{i}\right)\right]\right. \\
\left.+f_{c}^{4}\left[\exp \left[-\alpha f_{\tilde{c}}^{2}\right]-\sqrt{\pi \alpha f_{\tilde{c}}^{2}}\left(1-\operatorname{erf}\left[\sqrt{\alpha f_{\tilde{c}}^{2}}\right]\right)\right] H\left(f_{c}\right) S_{f}\left(f_{c}\right)\right\}, \\
\left\|\left.\boldsymbol{u}_{1 \mathrm{D}-\operatorname{dir}}^{S}\right|_{z=0}\right\| \approx \frac{16 \pi^{3}}{\mathrm{~g}}\left\{\left[\sum_{i=1}^{N_{f}} \Delta f_{i} H\left(f_{i}\right) f_{i}^{3} S_{f}\left(f_{i}\right)\right]+\frac{f_{c}^{5}}{f_{\tilde{c}}} H\left(f_{c}\right) S_{f}\left(f_{c}\right)\right\} .
\end{gathered}
$$

Appendix C.3. Unidirectional $1 D_{h}-S D$

For unidirectional approximations, $\|\boldsymbol{H}\|=1$ and the subsurface and surface unidirectional $1 \mathrm{D}_{h}$-SD numerical approximations simplify to

$$
\begin{aligned}
\left\|\boldsymbol{u}_{1 \mathrm{D} \text {-uni }}^{S}\right\| & \approx \frac{2 \pi \alpha}{|z|}\left\{\left[\sum_{i=1}^{N_{f}} \Delta f_{i} f_{i}^{3} \exp \left[-\alpha f_{i}^{2}\right] S_{f}\left(f_{i}\right)\right]\right. \\
& \left.+f_{c}^{4}\left[\exp \left[-\alpha f_{\tilde{c}}^{2}\right]-\sqrt{\pi \alpha f_{\tilde{c}}^{2}}\left(1-\operatorname{erf}\left[\sqrt{\alpha f_{\tilde{c}}^{2}}\right]\right)\right] S_{f}\left(f_{c}\right)\right\} \\
\left\|\left.\boldsymbol{u}_{1 \mathrm{D}-\text { uni }}^{S}\right|_{z=0}\right\| & \approx \frac{16 \pi^{3}}{\mathrm{~g}}\left\{\left[\sum_{i=1}^{N_{f}} \Delta f_{i} f_{i}^{3} S_{f}\left(f_{i}\right)\right]+\frac{f_{c}^{5}}{f_{\tilde{c}}} S_{f}\left(f_{c}\right)\right\} .
\end{aligned}
$$


Appendix C.4. Depth-integrated Stokes drift

The numerical depth-integrated Stokes drift approximations resemble their surface counterparts and only require small modifications. They are

$$
\begin{aligned}
\boldsymbol{u}_{2 \mathrm{D}}^{S \text {-int }} \approx 2 \pi & \sum_{j=1}^{N_{\theta}} \Delta \theta_{j}\left(\cos \theta_{j}, \sin \theta_{j}, 0\right) \\
\times & \left\{\left[\sum_{i=1}^{N_{f}} \Delta f_{i} f_{i} S_{f \theta}\left(f_{i}, \theta_{j}\right)\right]+\frac{f_{c}^{5}}{3 f_{\tilde{c}}^{3}} \mathcal{S}_{f \theta}\left(f_{c}, \theta_{j}\right)\right\}, \\
\left\|\boldsymbol{u}_{1 \mathrm{D} \text {-dir }}^{S \text {-int }}\right\| \approx 2 \pi & \left\{\left[\sum_{i=1}^{N_{f}} \Delta f_{i} H\left(f_{i}\right) f_{i} S_{f}\left(f_{i}\right)\right]+\frac{f_{c}^{5}}{3 f_{\tilde{c}}^{3}} H\left(f_{c}\right) S_{f}\left(f_{c}\right)\right\}, \\
\left\|\boldsymbol{u}_{1 \mathrm{D} \text {-uni }}^{S \text { Sint }}\right\| \approx 2 \pi & \left\{\left[\sum_{i=1}^{N_{f}} \Delta f_{i} f_{i} S_{f}\left(f_{i}\right)\right]+\frac{f_{c}^{5}}{3 f_{\tilde{c}}^{3}} S_{f}\left(f_{c}\right)\right\} .
\end{aligned}
$$

\section{Appendix D. Matlab Stokes drift functions}

Matlab functions for all six approximations (both depth-dependent and depth-integrated) can be found online at www . adreanwebb. com and the MATLAB Central File Exchange. 


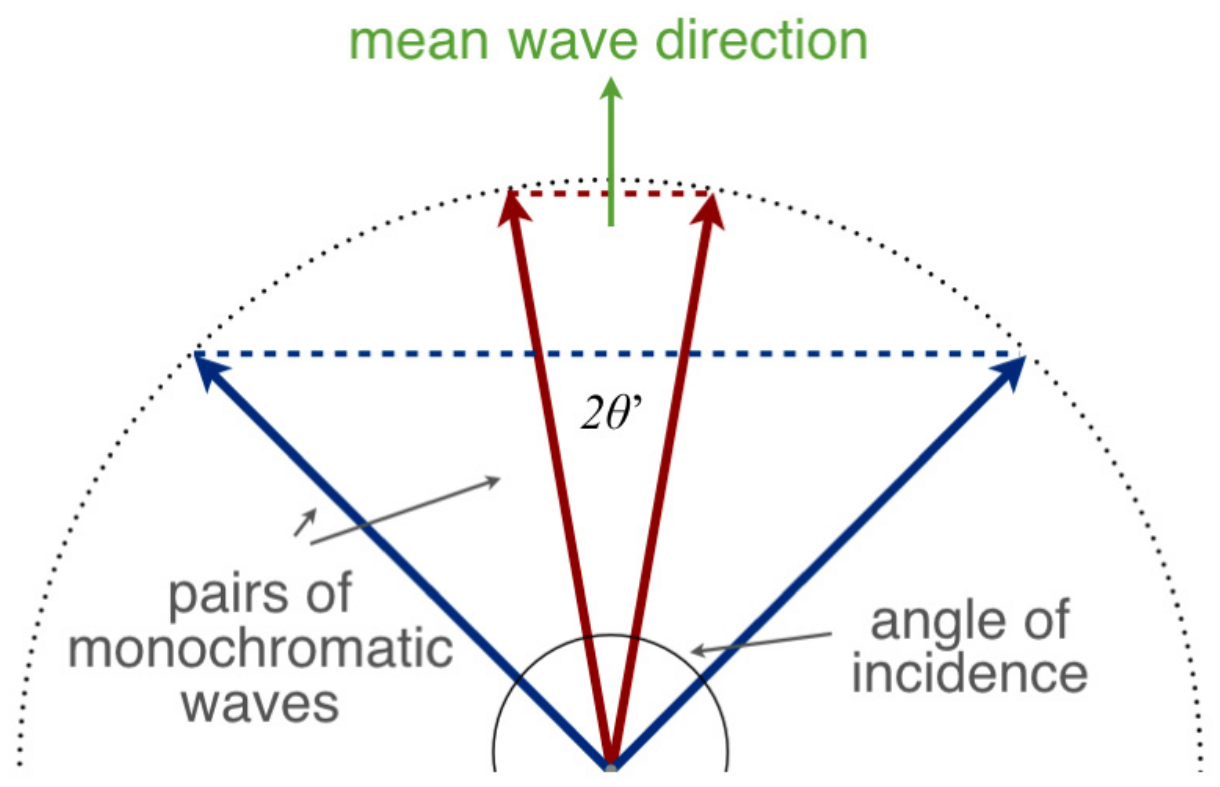

Figure 1: Here, pairs of monochromatic waves (red and blue) are shown traveling about a mean direction $\bar{\theta}=\pi / 2$ with a total directional difference (for each pair) of $2 \theta^{\prime}$. Only the $y$ vector components of the bichromatic waves contribute to Stokes drift. 


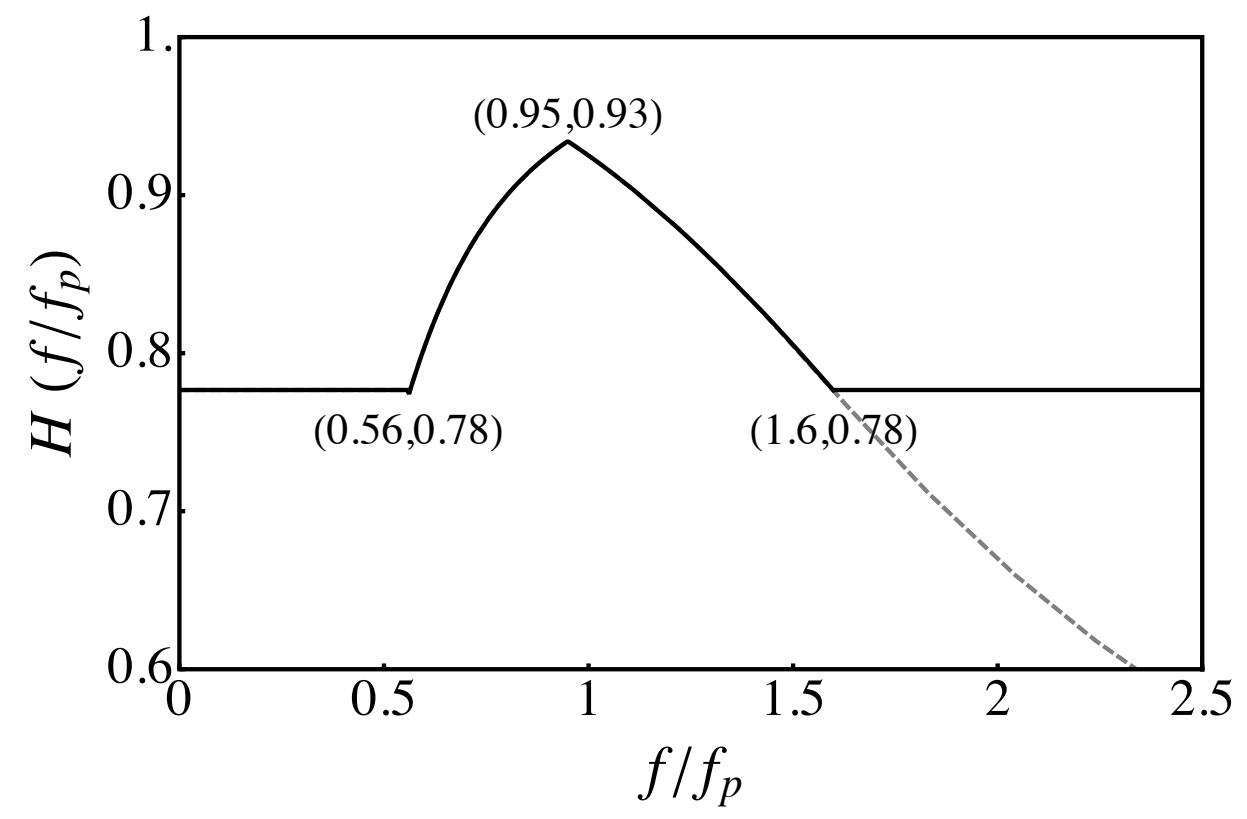

Figure 2: Magnitudes of the DHH (black solid) and DHH-B (gray dashed) directionalStokes-drift-components. 


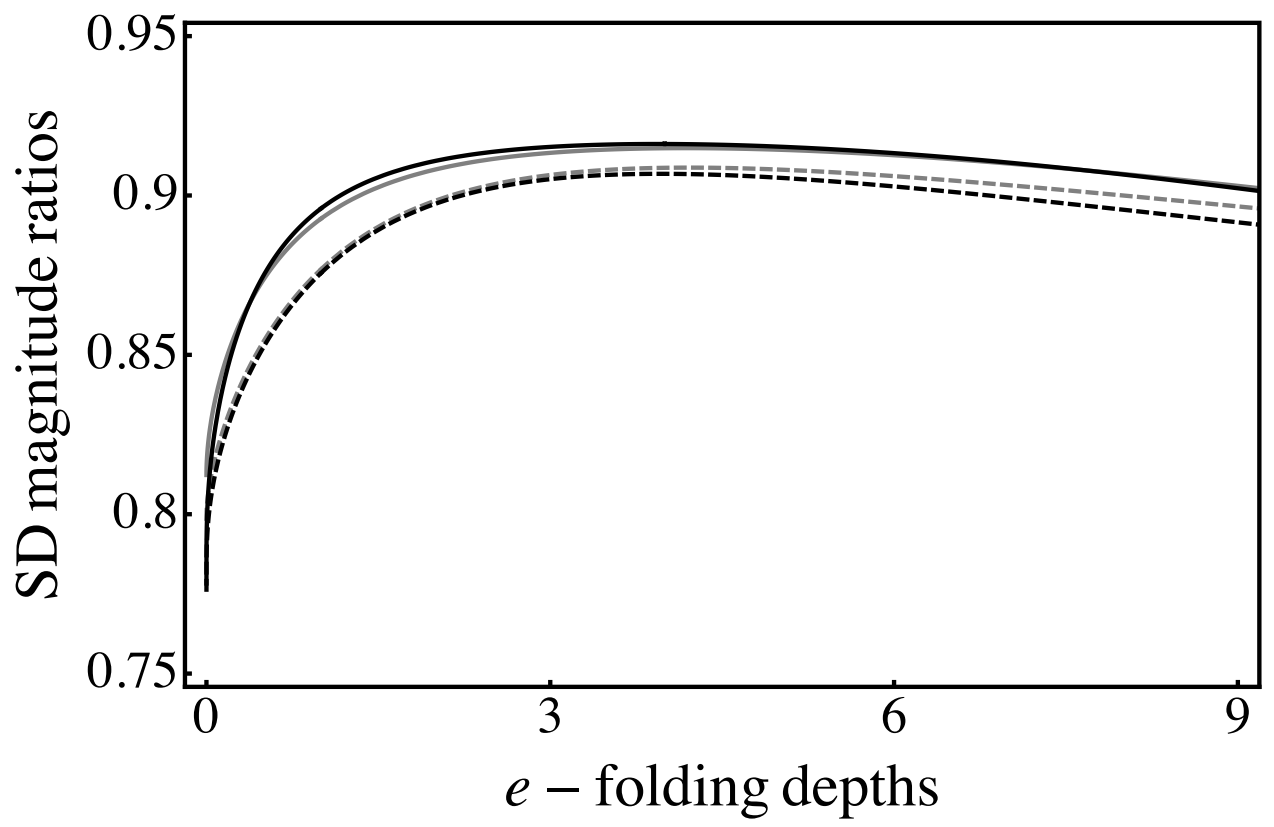

Figure 3: Ratios of $\mathbf{1} \mathbf{D}_{h}$-DHH-SD to $\mathbf{1} \mathbf{D}_{h}$-SD magnitudes using prescribed spectra for continuous $e$-folding depths: JONSWAP (gray solid), PM (gray dashed), fetch-limited DHH (black solid), and fully-developed DHH (black dashed). 


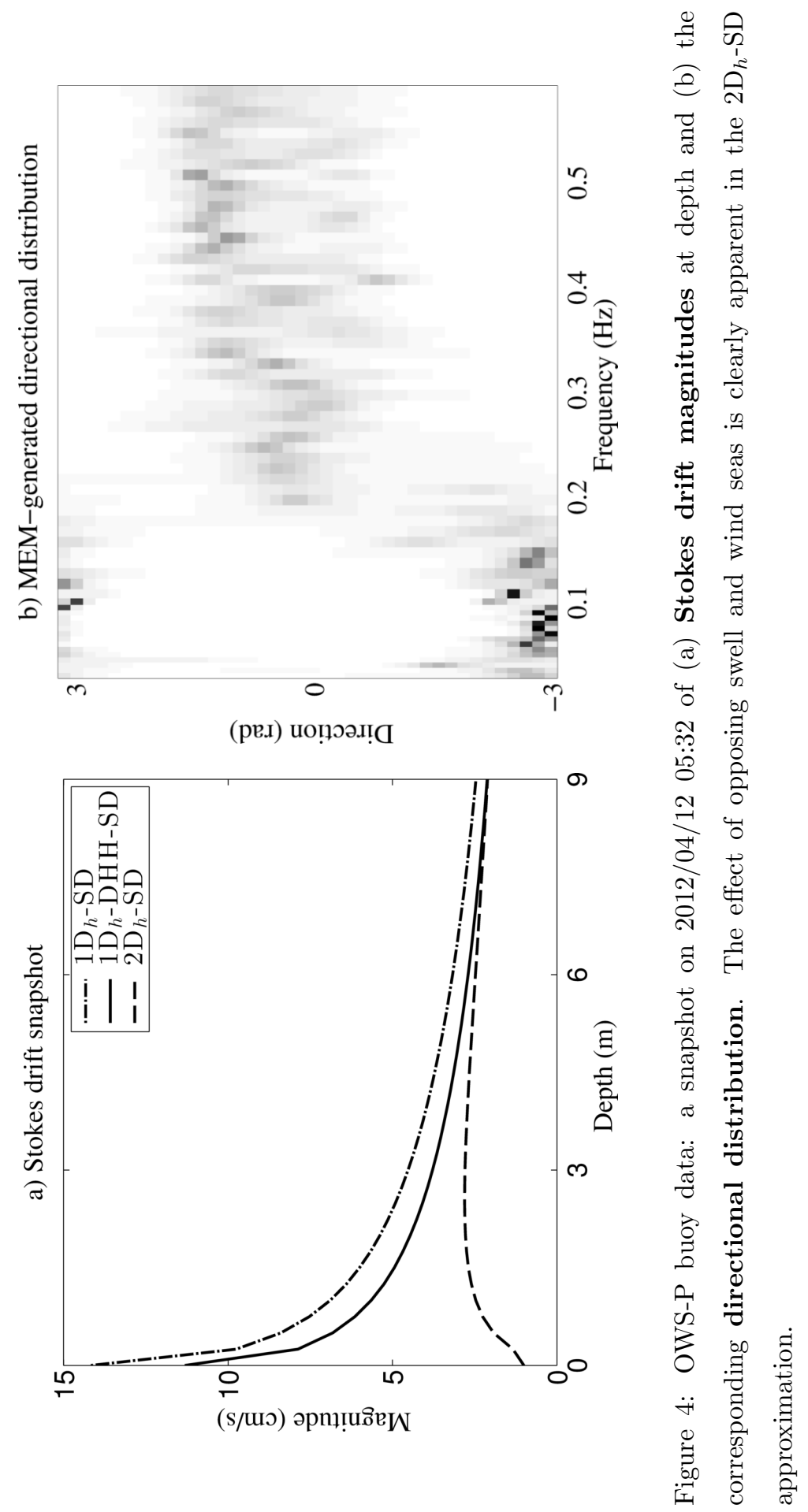




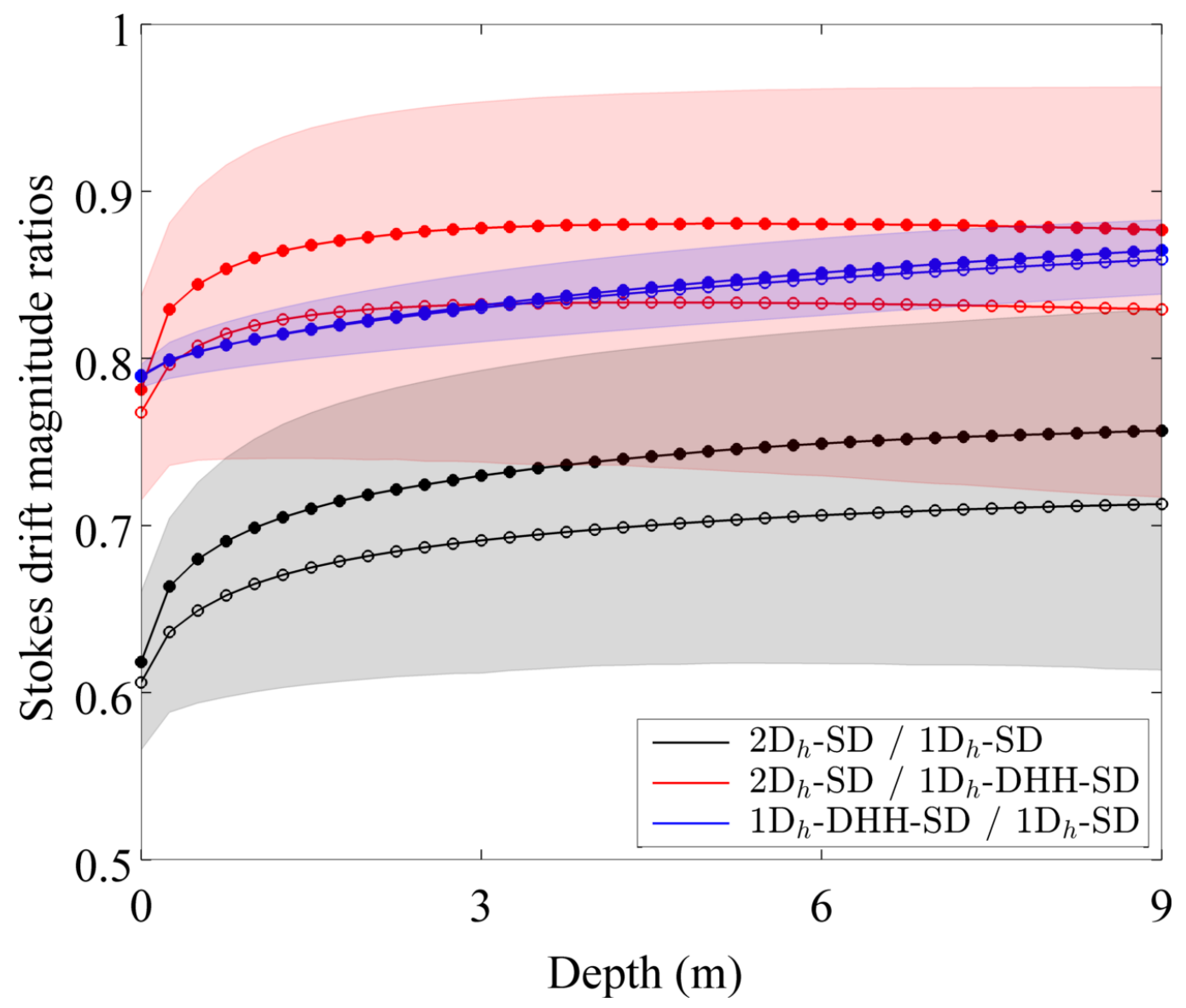

Figure 5: OWS-P buoy mean (open circles) and median (closed circles) Stokes drift magnitude ratios with the two-thirds centered distribution shaded for the 27 month period from 2010/06/21 to 2012/09/20. 


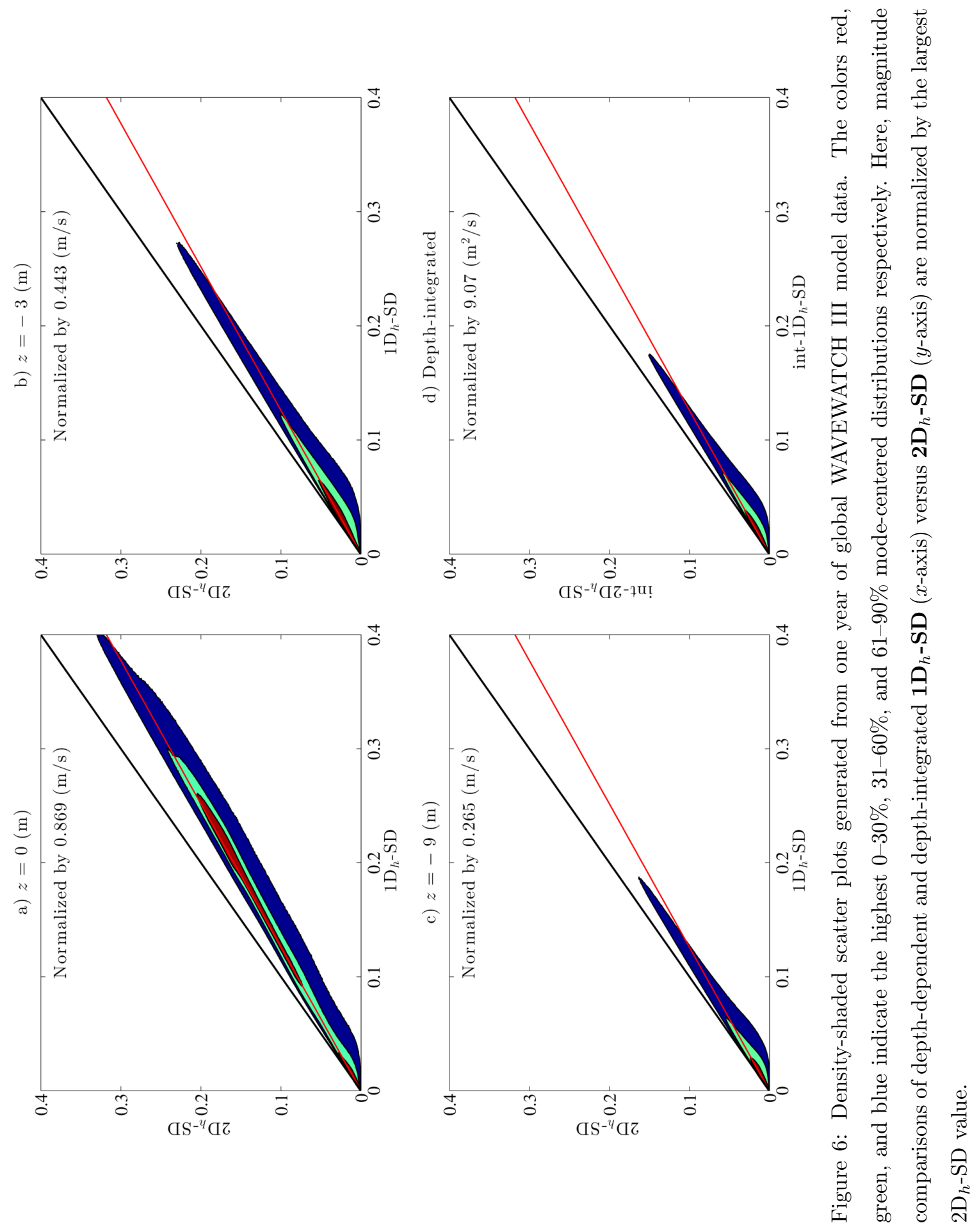




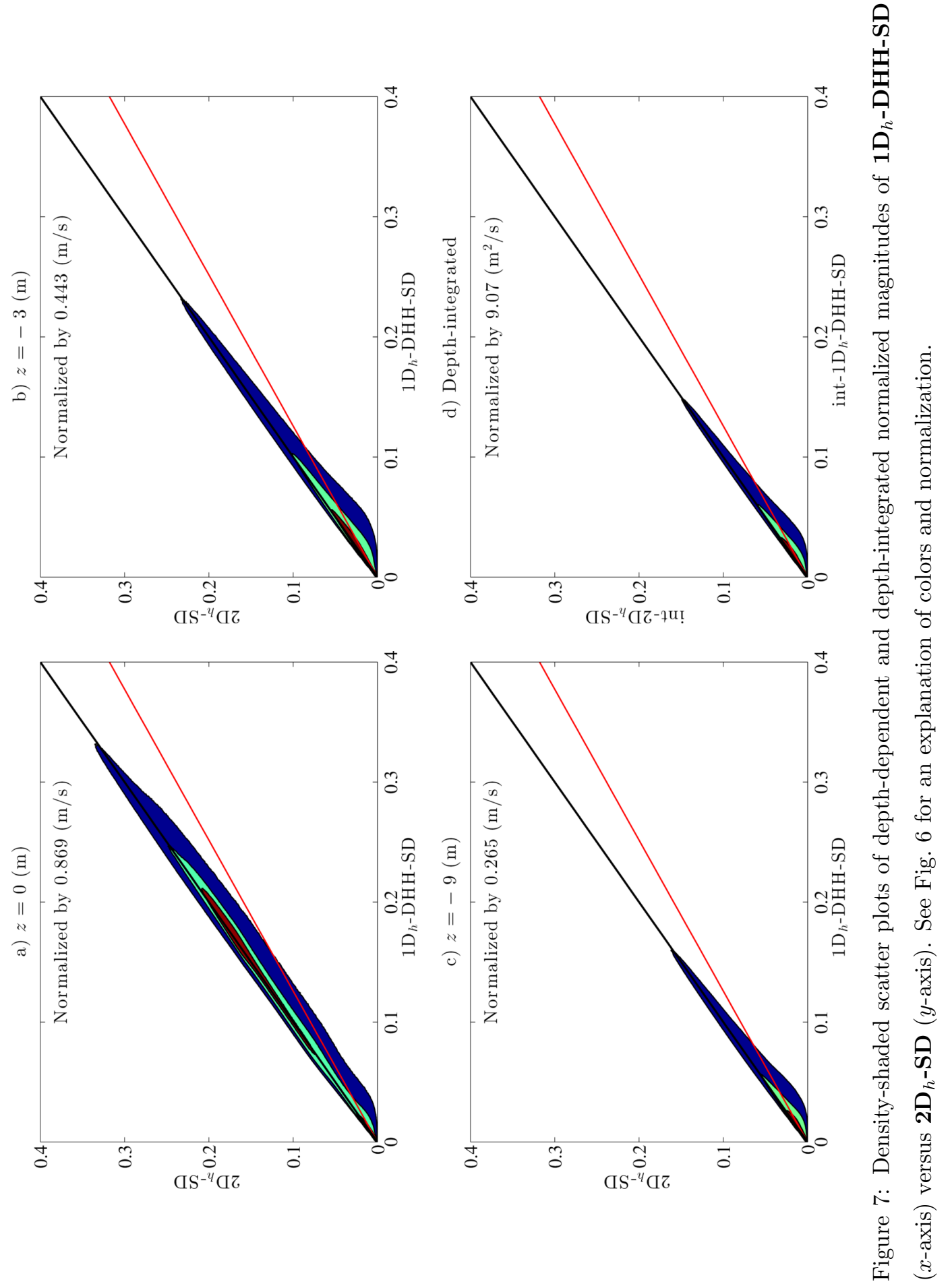




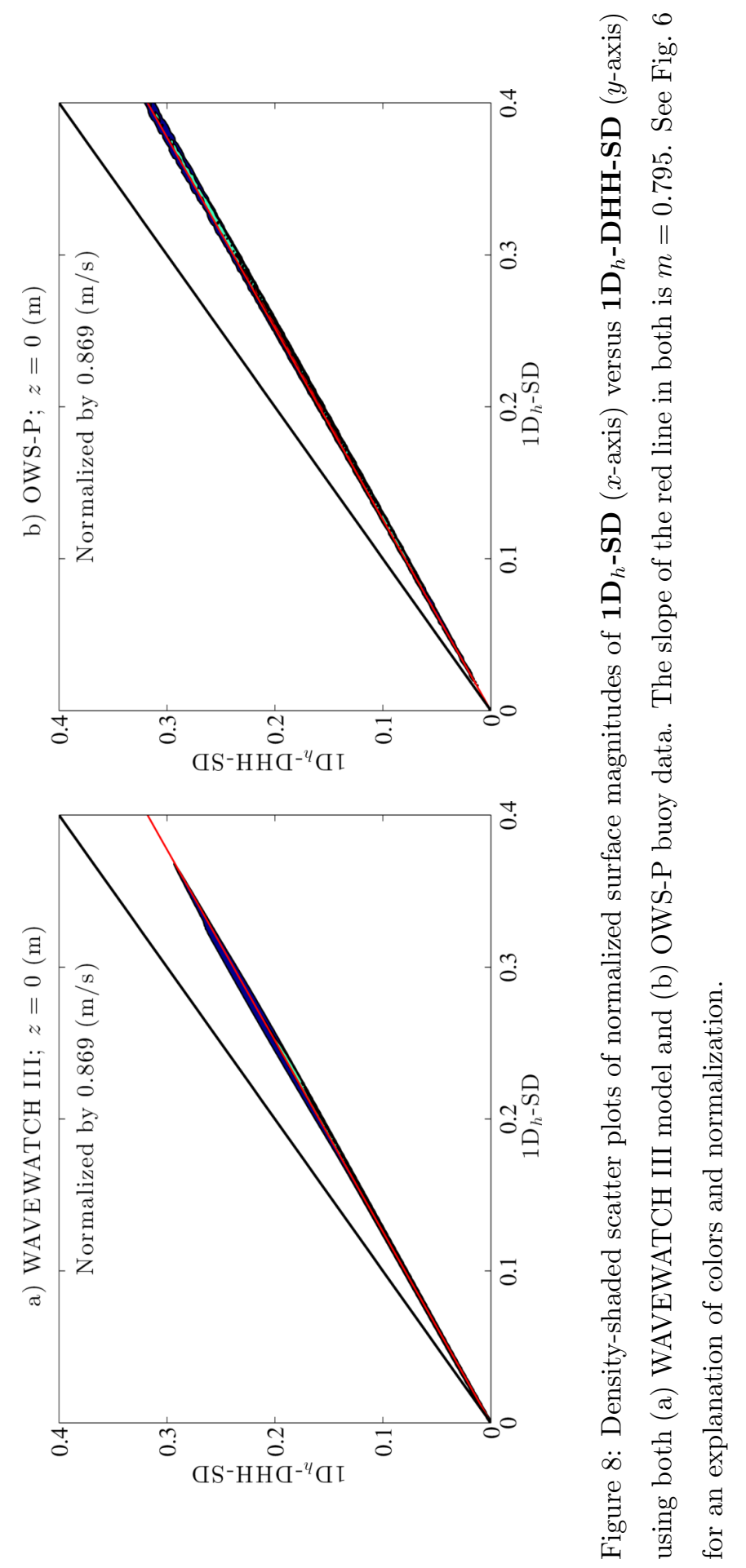




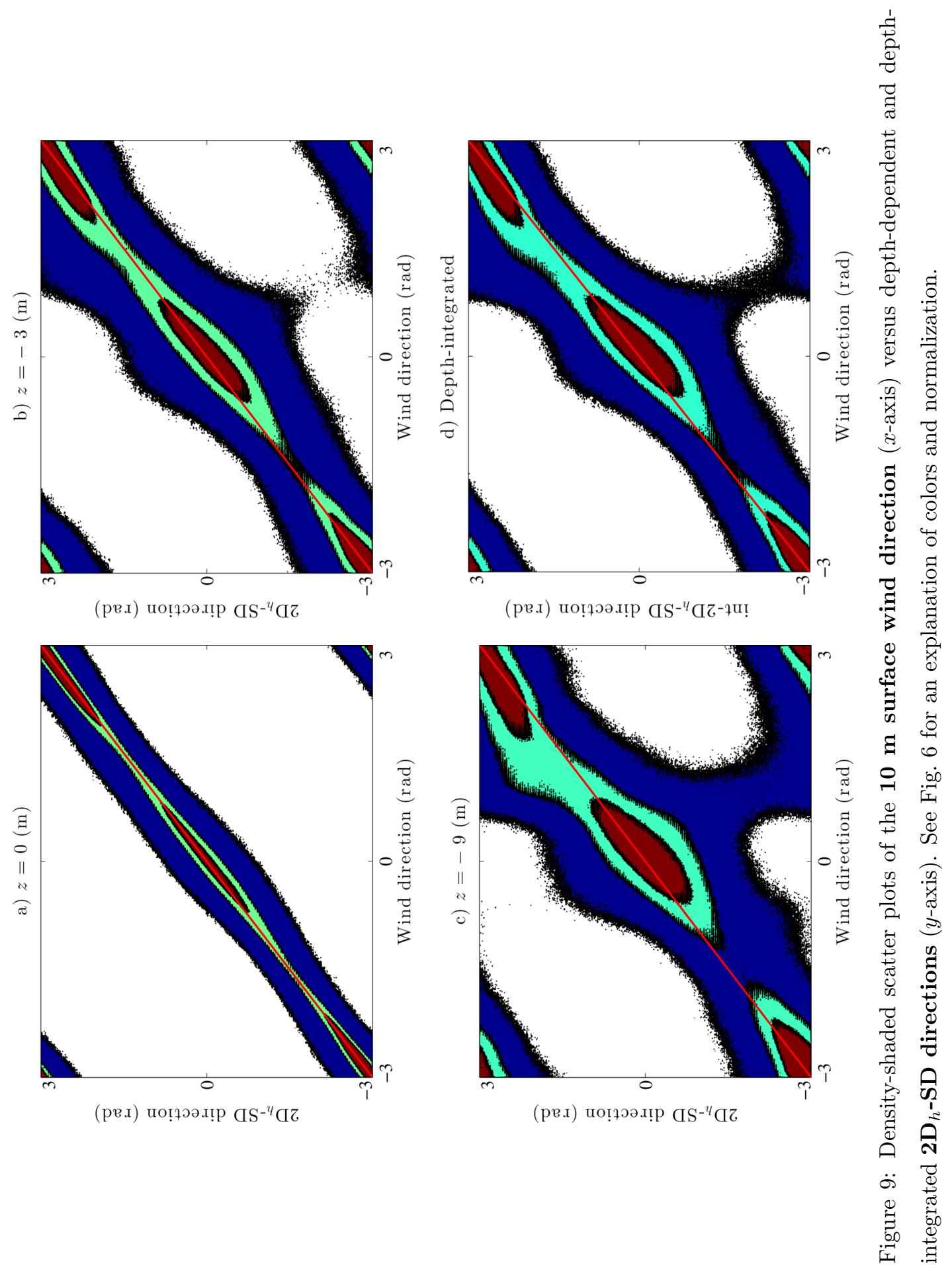



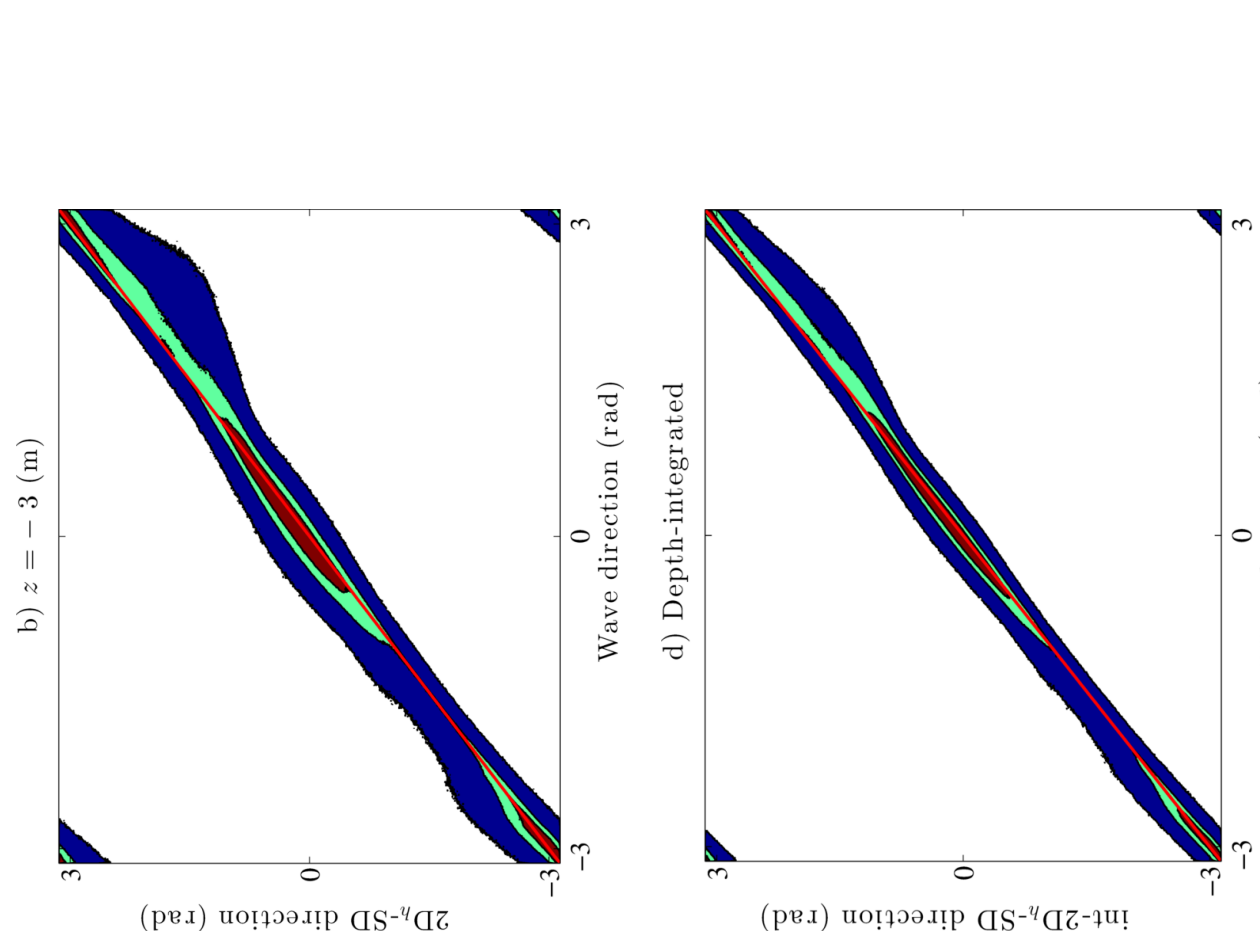

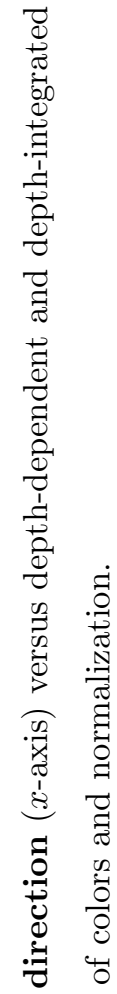
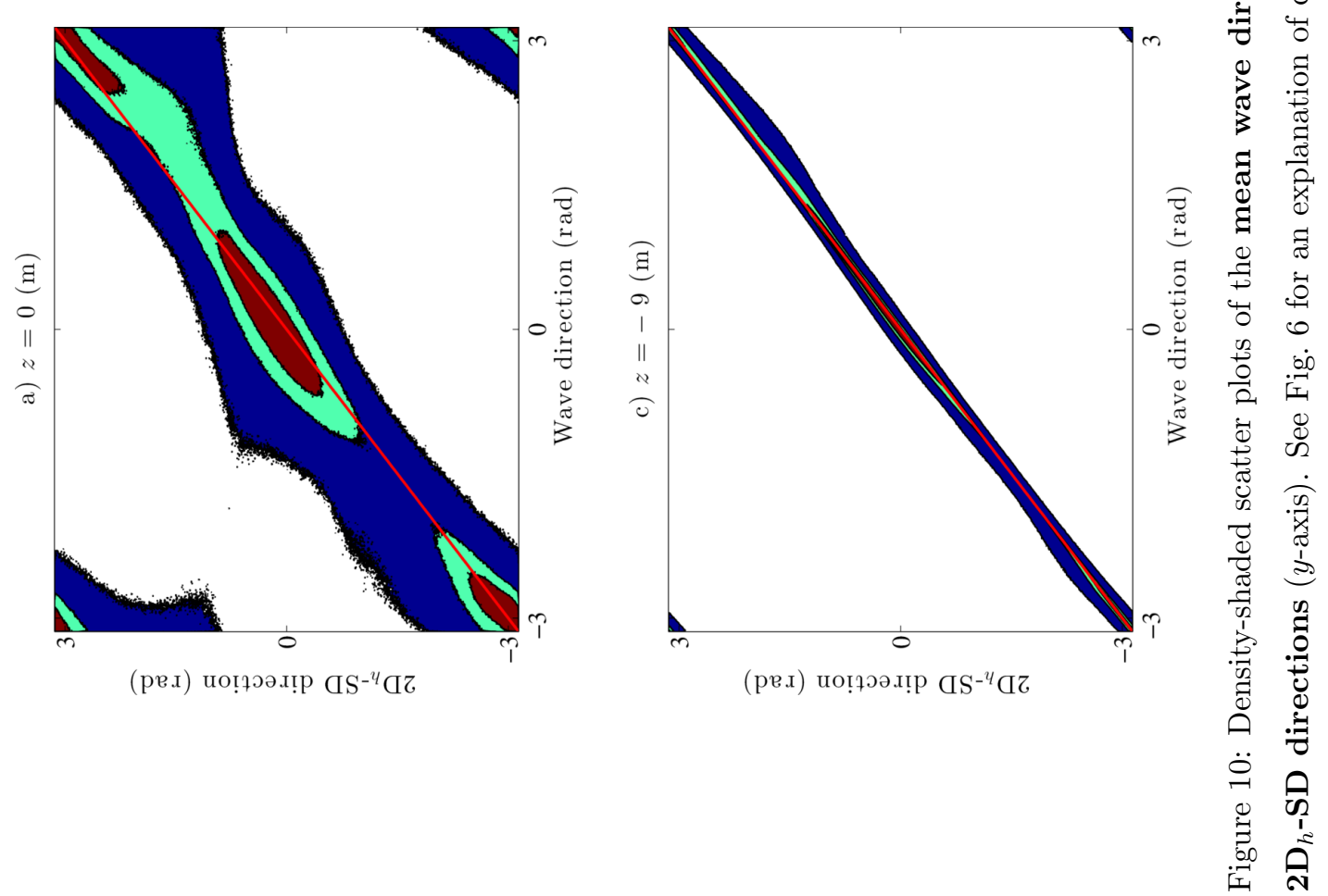


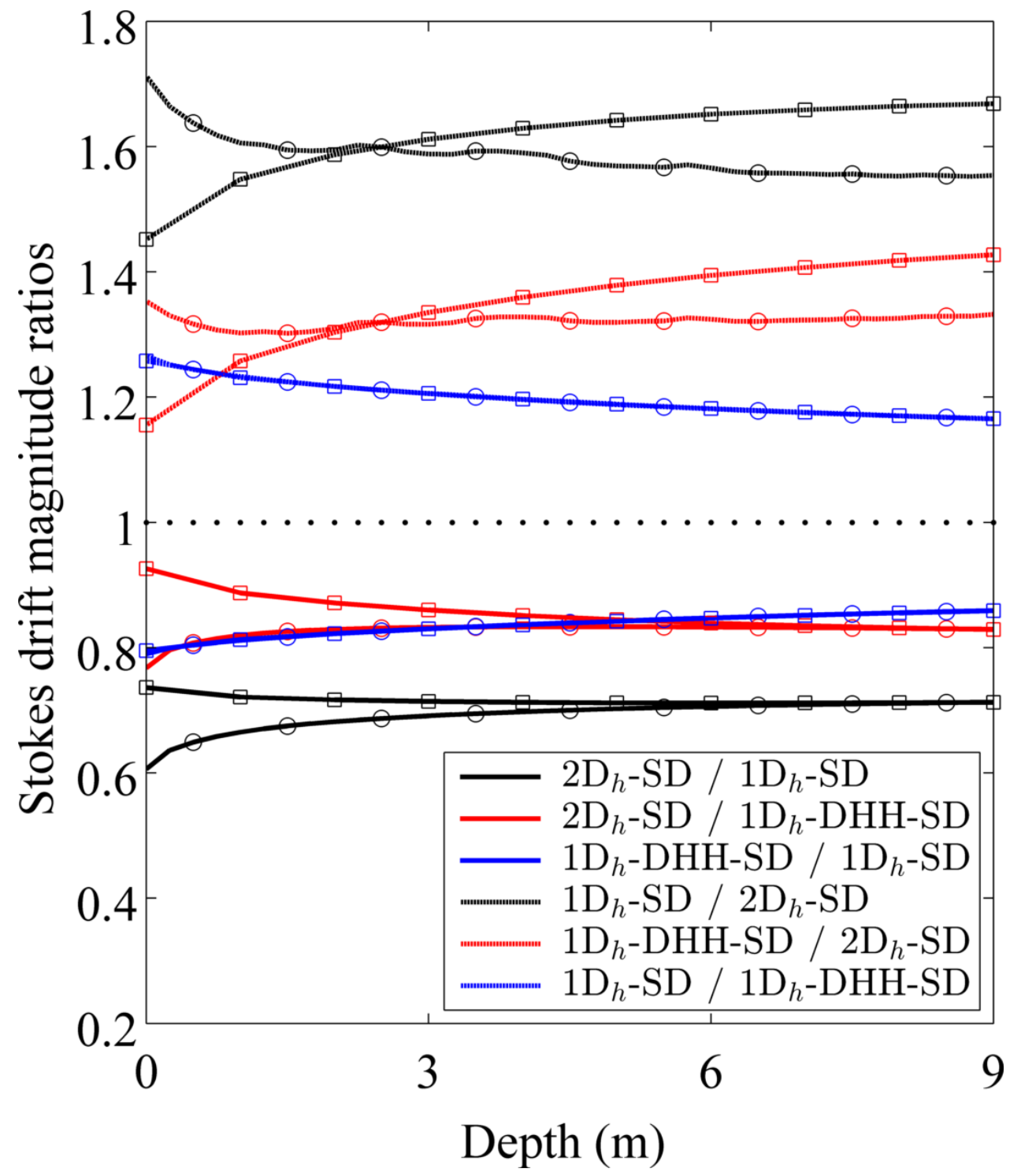

Figure 11: A comparison of mean magnitude ratios using OWS-P buoy (circles) and WAVEWATCH III model (squares) data. Physical processes are differentiated by color with blue for wave spreading $\left(1 \mathrm{D}_{h}\right.$-DHH-SD and $\left.1 \mathrm{D}_{h}-\mathrm{SD}\right)$, red for multidirectional wave $\left(2 \mathrm{D}_{h}\right.$-SD and $1 \mathrm{D}_{h}$-DHH-SD), and black for combined ( $2 \mathrm{D}_{h}$-SD and $1 \mathrm{D}_{h}$-SD) effects. In addition, lower and upper halves represent directional magnitude losses and overestimation errors respectively. 


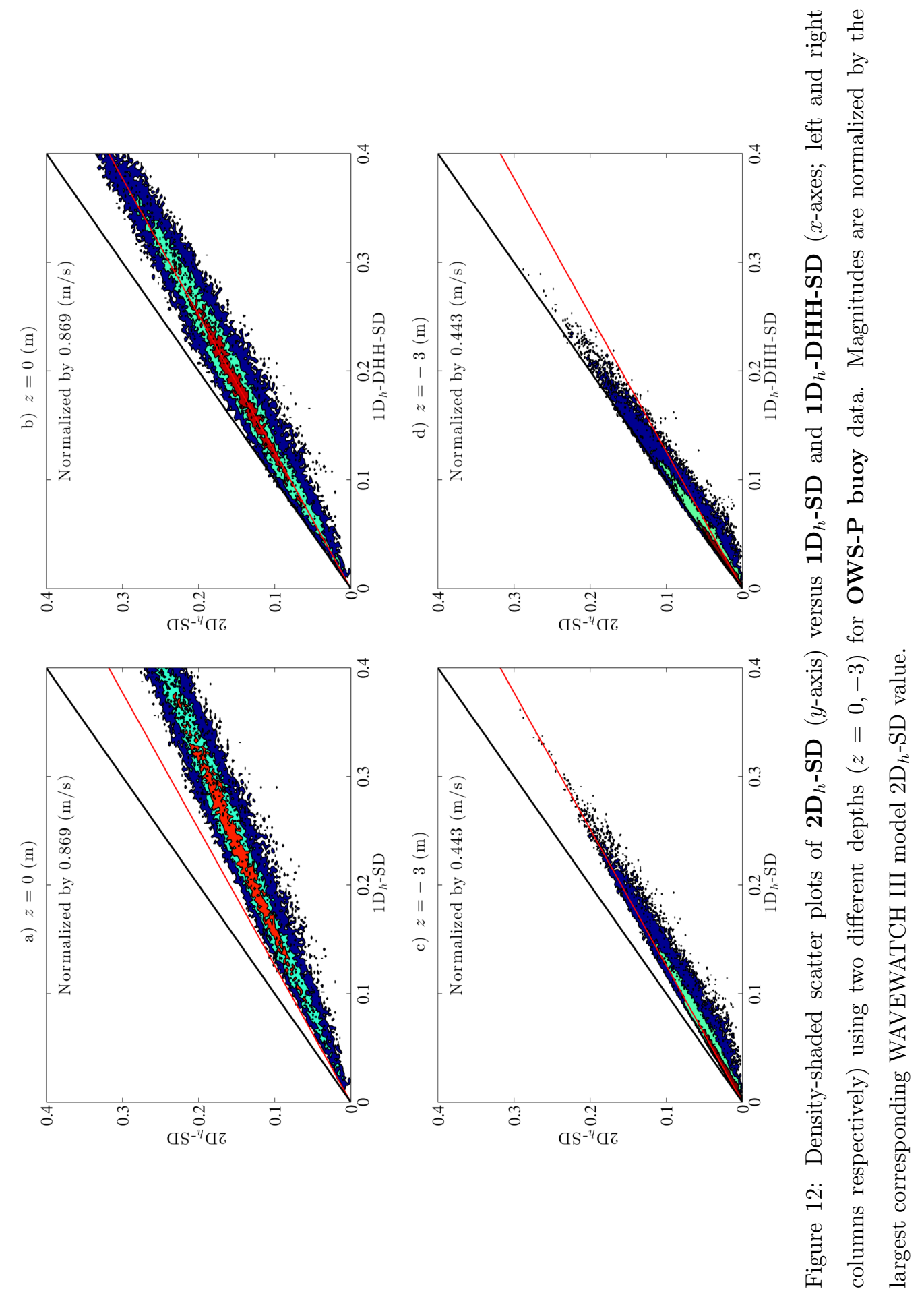


Table 1: Abbreviation guide for the random sea Stokes drift approximations

\begin{tabular}{ll}
\hline $1 \mathrm{D}_{h}, 2 \mathrm{D}_{h}$ & Horizontally one-, two-dimensional. \\
$2 \mathrm{D}_{h}-\mathrm{SD}$ & Directional random seas Stokes drift; \\
& uses 2D wave spectra. \\
$1 \mathrm{D}_{h}-\mathrm{SD}$ & Unidirectional random seas Stokes drift; \\
& uses 1D wave spectra. \\
$1 \mathrm{D}_{h}-\mathrm{DHH}-\mathrm{SD}$ & Uses 1D wave spectra with the Donelan \\
& et al. (1985) directional distribution to \\
& correct for wave spreading. \\
$1 \mathrm{D}_{h}$-DHH-B-SD & Uses 1D wave spectra with the Banner \\
& (1990) directional distribution to correct \\
& for wave spreading. \\
& Depth-integrated form of the depth- \\
int-*-SD & dependent approximations. \\
\hline
\end{tabular}


Table 2: Example $e$-folding depths $\left|z_{n}\right|$ (m) for the peak frequencies $f_{p}=0.05,0.16,0.34$ $(\mathrm{Hz})$. For reference, $\exp [-\{0.01,0.5,1,2,3\}]=\{0.990,0.607,0.368,0.135,0.050\}$.

\begin{tabular}{lllllll}
\hline$f_{p}$ & $T_{p}$ & $\left|z_{0.01}\right|$ & $\left|z_{0.5}\right|$ & $\left|z_{1}\right|$ & $\left|z_{2}\right|$ & $\left|z_{3}\right|$ \\
\hline 0.34 & 2.94 & 0.0107 & 0.537 & 1.07 & 2.15 & 3.22 \\
0.16 & 6.25 & 0.0485 & 2.43 & 4.85 & 9.71 & 14.6 \\
0.05 & 20 & 0.497 & 24.8 & 49.7 & 99.4 & 149 \\
\hline
\end{tabular}


Table 3: Ratio of $\mathbf{1} \mathbf{D}_{h}$-DHH-SD to $\mathbf{1} \mathbf{D}_{h}$-SD magnitudes using empirical spectra for various e-folding depths. Ratios with DHH1 (fetch-limited) and DHH2 (fully-developed) are not provided at $z_{0}$ since the spectra is undefined at the surface (see Section 4.1).

\begin{tabular}{lllllll}
\hline & $z_{0}$ & $z_{0.01}$ & $z_{0.5}$ & $z_{1}$ & $z_{2}$ & $z_{3}$ \\
\hline JONSWAP & 0.812 & 0.820 & 0.874 & 0.893 & 0.908 & 0.913 \\
DHH1 & - & 0.803 & 0.875 & 0.896 & 0.911 & 0.915 \\
PM & 0.799 & 0.805 & 0.854 & 0.877 & 0.898 & 0.906 \\
DHH2 & - & 0.795 & 0.853 & 0.876 & 0.897 & 0.905 \\
\hline
\end{tabular}


Table 4: A comparison of mean depth-integrated magnitude ratios using WAVEWATCH III model data. Mean magnitude losses and overestimation errors (mean reciprocal losses) are listed for wave spreading (int-1 $\mathrm{D}_{h}$-DHH-SD and int-1 $\mathrm{D}_{h}$-SD), multidirectional wave (int-2 $\mathrm{D}_{h}$-SD and int-1 $\mathrm{D}_{h}$-DHH-SD), and combined (int-2 $\mathrm{D}_{h}$-SD and int- $1 \mathrm{D}_{h}$-SD) effects.

\begin{tabular}{lll}
\hline & loss ratio & error ratio \\
\hline wave spreading & 0.841 & 1.191 \\
multidirectional wave & 0.830 & 1.410 \\
combined & 0.698 & 1.682 \\
\hline
\end{tabular}

